\title{
Antinorms on cones: duality and applications *
}

\author{
Vladimir Yu. Protasov ${ }^{\dagger}$
}

\begin{abstract}
An antinorm is a concave nonnegative homogeneous functional on a convex cone. It is shown that if the cone is polyhedral, then every antinorm has a unique continuous extension from the interior of the cone. The main facts of the duality theory in convex analysis, in particular, the Fenchel-Moreau theorem, are generalized to antinorms. However, it is shown that the duality relation for antinorms is discontinuous. In every dimension there are infinitely many self-dual antinorms on the positive orthant and, in particular, infinitely many autopolar polyhedra. For the two-dimensional case, we characterise them all. The classification in higher dimensions is left as an open problem. Applications to linear dynamical systems, to the Lyapunov exponent of random matrix products, to the lower spectral radius of nonnegative matrices, and to convex trigonometry are considered.

Keywords: linear operator, cone, nonnegative matrix, norm, concave functional, duality, self-duality, polar, autopolar, linear switching system, Lyapunov exponent, lower spectral radius, convex trigonometry
\end{abstract}

AMS 2000 Mathematical Subject classification: 46B20, 52A21, 93D20, 37H15

\section{Introduction}

Let $K$ be a convex cone in $\mathbb{R}^{d}$. We always assume that a cone is closed, nondegenerate, i.e. possesses a nonempty interior, and pointed, i.e., does not contain a straight line. Every ray in the cone starting at the apex is either a generatrix, if it lies on the boundary, or an interior ray otherwise.

Definition 1 An antinorm on a cone $K$ is a nonnegative, somewhere positive, concave homogeneous functional on $K$. An antinorm is called positive if it is strictly positive at all points $x \in K \backslash\{0\}$.

${ }^{*}$ This work is supported by the Russian Science Foundation under grant 20-11-20169

†Steklov Mathematical Institute of Russian Academy of Sciences, Moscow, Russia, e-mail: v-protassov@yandex.ru 
In most cases we assume that $K$ is the positive orthant $\mathbb{R}_{+}^{d}$, but all definitions will be given in the general case.

Antinorms are concave analogues of norms. However, they cannot be defined in the whole space because there are no concave positively homogeneous functions on $\mathbb{R}^{d}$. That is why, an antinorm is usually restricted to some cone. It follows from the concavity that an antinorm is strictly positive (i.e., does not vanish) in the interior of $K$. It can vanish at some points of the boundary $\partial K$, which makes a difference form norms. Another difference is that antinorms can be discontinuous. However, as we shall see in Section 2, for polyhedral cones, in particular, for $\mathbb{R}_{+}^{d}$, the whole theory can be reduced to continuous antinorms by using the concept of continuous extension. The most remarkable differences between norms and antinorms are in the duality theory, which is developed in Sections 3. The main concepts and the basic facts (duals and polars, Young's inequality, refrexivity of duality, etc.) are similar to those for dual norms. However, the duality relation can be discontinuous even for continuous antinorms (Theorem 3 in Section 3). Moreover, while a self-dual norm in $\mathbb{R}^{d}$ is unique (the Euclidean one), there exist infinitely many different self-dual antinorms in $\mathbb{R}_{+}^{d}$. In case $d=2$, we give their complete classification. On the other hand, there is only one symmetric self-dual antinorm in $\mathbb{R}_{+}^{2}$, which is bounded by a hyperbola. This issue is studied in Section 4. Generalizations of those results to higher dimensions are left as open problems in Section 5. In Section 6 we analyse applications of antinorms to positive linear switching systems, to asymptotics of random matrix products, to the lower spectral radius, and to convex trigonometry.

Related works. Generalizations of the notion of the norm such as pseudonorm, seminorm, etc. have been thoroughly studied in the literature. The concept of antinorm is usually understood according to Definition 1 , as a positively homogeneous functional (i.e., $f \geq 0$ and $f(\lambda \boldsymbol{x})=\lambda f(\boldsymbol{x})$ for all $\lambda \geq 0$ ) with the reverse triangle inequality. As a rule, an antinorm is defined on a cone or, more generally, is defined piecewise on a fan, which is a partition of a space into several cones with a common apex. Similarly antinorms were defined [30, 38] as piecewise concave Minkowski functionals of star sets. This definition was put to good use for special construction of probability distributions [38. Matrix antinorms on the cone of positive definite symmetric matrices $\mathbb{M}_{d}$ were studied in [5, 6], where some important ineqialities for operator means were extended to antinorms. Again, the antinorm was defined as a positively homogeneous concave function (of a matrix). In particular, the Minkowski antinorm $f(A)=(\operatorname{det} A)^{1 / d}$ and the Schatten $q$-antinorms $f(A)=(\operatorname{tr} A)^{1 / q}, q \in(-\infty, 1]$, on the set $\mathbb{M}_{d}$ were considered in those works. The concept of antinorm was extended to von Neumann algebras [7]. On the other hands, in some works the term "antinorm" has a different meaning. For example, 23] a dual norm is called antinorm. In that work dual norms were analysed in context of the Radon curves and of the Minkowski content

We use the notion of antinorm according to Definition 1 as it is done in most of the literature. To the best of our knowledge, this concept originated with Merikoski [24] and was studied in [25, 26]. Independently and later antinorms were defined in aforementioned works. It was shown [34 that every i.i.d. sequence of random non-negative matrices, under some mild assumptions, possesses an invariant antinorm on the positive orthant. This result was applied for the problem of computation of the largest Lyapunov exponent for random 
matrices [18. Invariant antinorms were also exploited for computation of the lower spectral radius of matrix families [14, 15, 16]. The work [16] also analyses many properties of antinorms, including the basic facts of their duality. The antinorms were used in the stability theory for positive linear dynamical systems [13].

Remark 1 Since a positive homogeneous function cannot be concave on the whole space $\mathbb{R}^{d}$, antinorms are usually defined on convex cones. For example, they are often considered on the positive orthant $\mathbb{R}_{+}^{d}$ or, in case of matrix antinorms, in the cone of positive semitefinite matrices [5, 6]. In some works antinorms are defined on space $\mathbb{R}^{d}$, but in this case the whole space is split to several cones (forming a fan) and the antinorm is concave on each cone separately. An equivalent definition uses Minkowski functionals of star sets [30, 38].

Notation. For an antinorm $f$ on $K$, one defines its antiball $G=\{\boldsymbol{x} \in K \mid f(\boldsymbol{x}) \geq 1\}$ and an antisphere $\mathcal{S}=\{\boldsymbol{x} \in K \mid f(\boldsymbol{x})=1\}$. The antiball is convex and unbounded. Moreover, it is a conic body according to the following definition:

Definition $2 A$ conic body is a convex closed subset of a cone $K$ that does not contain the apex and possesses the following property: every interior ray of $K$ intersects that set by a ray. A conic polyhedron is a conic body defined in $K$ by a system of finitely many linear inequalities.

Clearly, all conic bodies are unbounded. Unlike usual polyhedra, a conic polyhedron can have only one vertex, for example $G=\left\{x \in \mathbb{R}_{+}^{d} \mid x_{i} \geq 1, i=1, \ldots, d\right\}$.

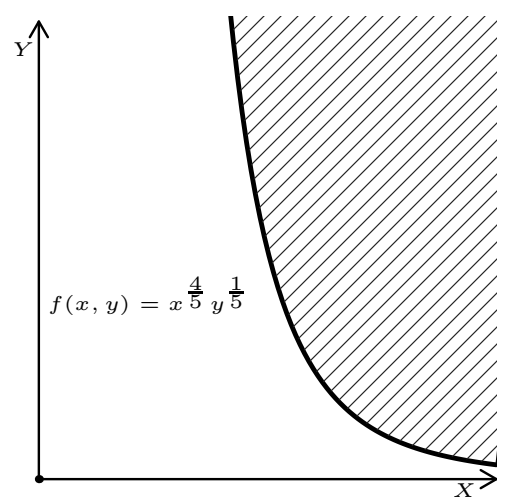

a)

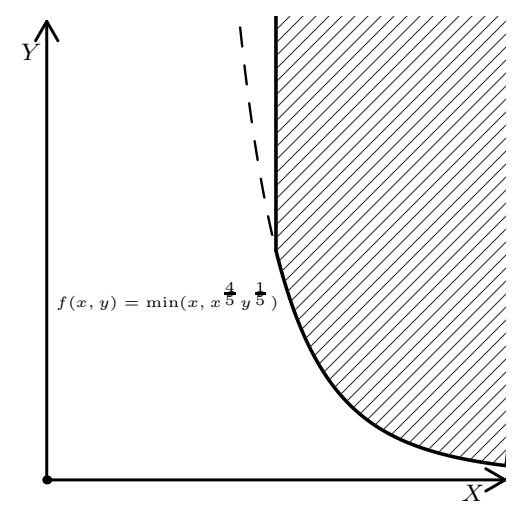

b)

Figure 1: The unit antiballs of the antinorms $x^{\frac{4}{5}} y^{\frac{1}{5}}$ (left) and $\min \left\{x^{\frac{4}{5}} y^{\frac{1}{5}}, x\right\}$ (right).

There is a one-to-one correspondence between antinorms and conic bodies. The antiball of an antinorm $f$ is a conic body. Conversely, every conic body $G$ defines a unique antinorm, similarly to the Minkowski functional: $f(\boldsymbol{x})=\sup \left\{\lambda>0 \mid \lambda^{-1} \boldsymbol{x} \in G\right\}$. The dual antinorm corresponds to the polar conic body. There are infinitely many autopolar conic polygons in $\mathbb{R}_{+}^{2}$. In Section 4 we explicitly classify them all. We are not aware of any example of autopolar polyhedra in $\mathbb{R}_{+}^{d}$ for $d \geq 3$ except for those reduced to two-dimensional ones. 
In what follows we usually drop the prefix "anti" is use the simple terminology "ball" in "sphere" for antinorms, when it does not lead to confusions. Fig. 1 shows the unit balls of two antinorms.

The vectors are denoted by bold letters and the scalars are denoted by usual letters, so $\boldsymbol{x}=\left(x_{1}, \ldots, x_{d}\right)$. We use the standard nonation $\mathbb{R}_{+}^{d}$ for the positive orthant, which consists of points with all coordinates being nonnegative; $\boldsymbol{x} \geq 0$ if $\boldsymbol{x} \in \mathbb{R}_{+}^{d}$ and $\boldsymbol{x}>0$ if $\boldsymbol{x} \in$ int $\mathbb{R}_{+}^{d}$; $\boldsymbol{x} \geq \boldsymbol{y}$ means that $\boldsymbol{x}-\boldsymbol{y} \geq 0$.

As usual, int $G, \partial G$, and co $G$ denotes respectively the interior, the boundary, and the convex hull of a set $G$. A convex body is a convex compact set with a nonempty interior. We denote the Euclidean norm in $\mathbb{R}^{d}$ by $|\cdot|$ and an arbitrary norm by $\|\cdot\|$. By $B_{r}(\boldsymbol{x})$ we denote the Euclidean ball of radius $r$ centered at $\boldsymbol{x}$. An inverse point to a given point $A \in \mathbb{R}^{d}$ is the image of $A$ under the inversion about the unit sphere centered at the origin. Thus, $A^{\prime}$ is inverse to $A$ if the vectors $O A$ and $O A^{\prime}$ are co-directed and $\left|O A^{\prime}\right| \cdot|O A|=1$.

\section{Continuity of antinorms}

It is well known that antinorms may be discontinuous. For example, the following antinorm in $\mathbb{R}_{+}^{2}: f(x, y)=x+y$ if $x>0$ and $f(x, y)=0$ if $x=0$, is discontinuous. The continuity issue is extremely important in generalizing many facts of convex analysis to antinorms. For example, the main duality result $f^{* *}=f$ is true provided an antinorm is continuous and may fail otherwise [16]. Moreover, continuity of antinorms is crucial in many applications. For instance, extremal and invariant antinorms of linear dynamical systems must be continuous to estimate the growth of trajectories, see Section 6 .

In case of antinorms on the positive cone $\mathbb{R}_{+}^{d}$, or, more generally, on a polyhedral cone, the whole theory can be restricted to the continuous case. To see this we need to establish some properties of concave functions defined on polyhedral sets.

A convex closed set $G \subset \mathbb{R}^{d}$ with a nonempty interior is called polyhedral if it is a set of solutions of a system of linear inequalities. A bounded polyhedral set is a polyhedron.

Since a concave function is continuous at any interior point of its domain, it follows that the discontinuity can occur only on the boundary. Let $f$ be a non-negative concave function defined on the interior of a convex set $G$. We extend it onto the boundary of $G$ by the limit:

$$
F(\boldsymbol{x})=\lim _{\substack{\boldsymbol{y} \in \text { int } G \\|\boldsymbol{x}-\boldsymbol{y}| \rightarrow 0}} f(\boldsymbol{y}), \quad \boldsymbol{x} \in \partial G
$$

Lemma 1 If $G$ is a polyhedral set, then for every point $\boldsymbol{x} \in \partial G$, the limit (1) is well-defined.

Proof. Assume the contrary. In this case there are two numbers $a, b$ such that $a<b$ and two sequences $\left\{\boldsymbol{y}_{k}\right\}_{k \in \mathbb{N}},\left\{\boldsymbol{z}_{k}\right\}_{k \in \mathbb{N}}$ of points from int $G$ that converge to $\boldsymbol{x}$ and such that $f\left(\boldsymbol{y}_{k}\right)<a<b<f\left(\boldsymbol{z}_{k}\right)$ for all $k \in \mathbb{N}$. Take a number $r$ so small that the ball $B_{r}(\boldsymbol{x})$ does not intersect other face planes of $G$ than those containing $\boldsymbol{x}$. Denote by $M$ the supremum of the function $f$ in the interior of this ball. The concavity implies that $M<+\infty$. Let 
$\varepsilon<\frac{r(b-a)}{M}$. For every point $\boldsymbol{z} \in B_{\varepsilon}(\boldsymbol{x}) \cap G$, the point $\boldsymbol{x}+\frac{r}{\varepsilon}(\boldsymbol{z}-\boldsymbol{x})$ belongs to $B_{r}(\boldsymbol{x}) \cap G$. Now take arbitrary point $\boldsymbol{z}_{k} \in B_{\varepsilon}(\boldsymbol{x}) \cap G$ and a point $\boldsymbol{y}_{n}$ very close to $\boldsymbol{x}$. Then the point $\boldsymbol{a}=\boldsymbol{x}+\frac{r}{\varepsilon}\left(\boldsymbol{z}_{k}-\boldsymbol{y}_{n}\right)$ belongs to $B_{r}(\boldsymbol{x}) \cap G$. Therefore $f(\boldsymbol{a}) \leq M$. On the other hand, the concavity yields $f(\boldsymbol{a})>f(\boldsymbol{x})+\frac{r}{\varepsilon}(b-a)>f(\boldsymbol{x})+M \geq M$. Thus, $f(\boldsymbol{a})>M$, which is a contradiction.

Remark 2 Lemma 1 may fail for non-polyhedral domains as the following example demonstrates.

Example 1 Consider a disc $G \subset \mathbb{R}^{2}$ of radius 1 centered at the point $(1,0)$ and a function $f$ whose graph in $\mathbb{R}^{3}$ is a convex hull of this disc and of the point $(0,0,1)$, see Fig. 2. Take an arbitrary sequence of points $\boldsymbol{x}_{k}$ on the boundary of $G$ which converges to the point $\boldsymbol{x}_{0}=(0,0)$. Then, obviously, both sequences $\frac{1}{2} \boldsymbol{x}_{k}$ and $\frac{1}{3} \boldsymbol{x}_{k}$ converge to $\boldsymbol{x}_{0}$. However, $\lim _{k \rightarrow \infty} f\left(\frac{1}{2} \boldsymbol{x}_{k}\right)=\frac{1}{2}$, while $\lim _{k \rightarrow \infty} f\left(\frac{1}{3} \boldsymbol{x}_{k}\right)=\frac{2}{3}$.

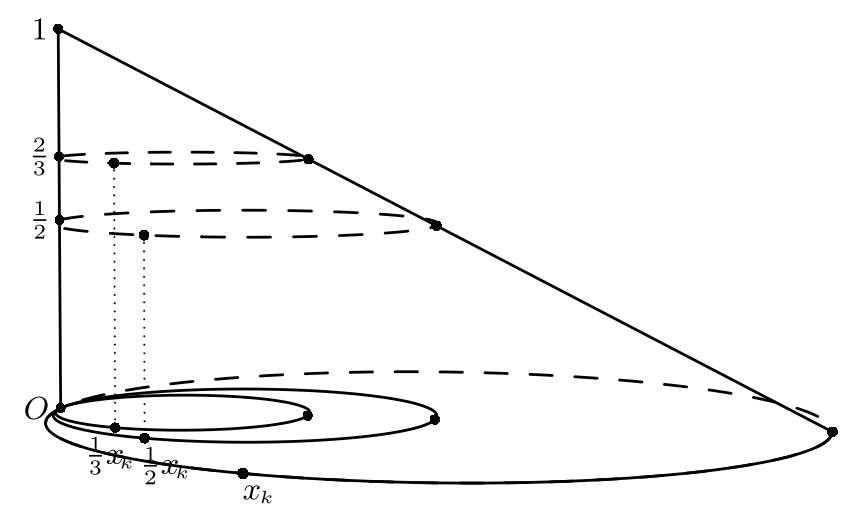

Figure 2: A concave function without continuous extension

The same construction provides a counterexample to Lemma 1 for every non-polyhedral domain. If a domain $G \subset \mathbb{R}^{d}$ is not polyhedral, then there exist points $\left\{\boldsymbol{x}_{k}\right\}_{k=0}^{\infty}$ on its boundary such that $\boldsymbol{x}_{k} \rightarrow \boldsymbol{x}_{0}$ as $k \rightarrow \infty$ and for every $k \geq 1$, the open interval between $\boldsymbol{x}_{0}$ and $\boldsymbol{x}_{k}$ lies in the interior of $G$. Then we place $G$ in the coordinate subspace of $\mathbb{R}^{d+1}$ spanned by the first $d$ basis vectors so that $\boldsymbol{x}_{0}$ coincides with the origin and consider the function $f$ on $G$ whose graph is the convex hull of $G$ and of the point $(0, \ldots, 0,1)$. Then we argue as for the case when $G$ is the disc.

Definition 3 Let $f$ be a nonnegative concave function defined on a convex closed set $G$ with a nonempty interior. A continuous concave function $F$ on $G$ such that $F(\boldsymbol{x})=f(\boldsymbol{x})$ for all $\boldsymbol{x} \in \operatorname{int} G$ is called a continuous extension of $f$.

Not every nonnegative concave function possesses a continuous extension. Nevertheless, if the set $G$ is polyhedral, then such an extension does exist. Moreover, it is unique and majorizes the function $f$. 
Proposition 1 Every nonnegative concave function $f$ on a polyhedral set $G$ possesses a unique continuous extension $F$. That extension is defined on the boundary of $G$ by formula (1). For every $\boldsymbol{x} \in G$, we have $F(\boldsymbol{x}) \geq f(\boldsymbol{x})$.

Proof. On $\operatorname{int} G$ we have $F=f$. At every boundary point the function $F$ is welldefined by (1), as it follows from Lemma 1. Continuity of $F$ is easily proved by assuming the contrary. Let $F$ be discontinuous at some point $\boldsymbol{x} \in G$. Since a concave function is continuous on the interior of the domain, it follows that $\boldsymbol{x} \in \partial G$, hence $F(\boldsymbol{x})$ is defined by formula (1). There exists a sequence $\left\{\boldsymbol{x}_{k}\right\}_{k \in \mathbb{N}}$ of points from $G$, which converges to $\boldsymbol{x}$ but $f\left(\boldsymbol{x}_{k}\right)$ does not converge to $F(\boldsymbol{x})$. Define a new sequence $\left\{\boldsymbol{y}_{k}\right\}_{k \in \mathbb{N}}$ as follows: if $\boldsymbol{x}_{k} \in \operatorname{int} G$, then $\boldsymbol{y}_{k}=\boldsymbol{x}_{k}$, otherwise $\boldsymbol{y}_{k}$ is a point from int $G$ close to $\boldsymbol{x}_{k}$ for which $f\left(\boldsymbol{y}_{k}\right)$ is close to $F\left(\boldsymbol{x}_{k}\right)$ (such points exist due to formula (1)). Then $\boldsymbol{y}_{k} \rightarrow \boldsymbol{x}$, but $f\left(\boldsymbol{y}_{k}\right)$ does not converge to $F(\boldsymbol{x})$, which contradicts to the definition of $F(\boldsymbol{x})$.

To prove that $F(\boldsymbol{x}) \geq f(\boldsymbol{x})$, we take an arbitrary point $\boldsymbol{y} \in \operatorname{int} G$, in which $F(\boldsymbol{y})=f(\boldsymbol{y})$. Concavity implies that

$$
(1-t) f(\boldsymbol{x}) \leq f((1-t) \boldsymbol{x}+t \boldsymbol{y})-t f(\boldsymbol{y})=F((1-t) \boldsymbol{x}+t \boldsymbol{y})-t F(\boldsymbol{y}) .
$$

Taking limit as $t \rightarrow+0$, we have $F((1-t) \boldsymbol{x}+t \boldsymbol{y}) \rightarrow F(\boldsymbol{x})$ by continuity, and hence $f(\boldsymbol{x}) \leq F(\boldsymbol{x})$.

If $f$ is continuous, then $F \equiv f$. According to Proposition 1, all concave functions on $G$ are obtained from some continuous concave function by reducing its values on the boundary of $G$. They can be arbitrarily reduced at extreme points, and then reduced in all other points of the boundary to keep the function concave.

Corollary 1 Every antinorm $f$ on $\mathbb{R}_{+}^{d}$ possesses a unique continuous extension $F$. At every boundary point, we have $F(\boldsymbol{x}) \geq f(\boldsymbol{x})$.

Thus, we have proved the following classification of antinorms on $\mathbb{R}_{+}^{d}$.

Theorem 1 Every antinorm in $\mathbb{R}_{+}^{d}$ is obtained from a continuous antinorm by arbitrary reducing its values on the boundary that keeps the function concave and homogeneous on all coordinate subspaces.

Example 2 The antinorm $f: \mathbb{R}_{+}^{3} \rightarrow \mathbb{R}_{+}$defined by the formula

$$
f(\boldsymbol{x})= \begin{cases}\left(\sqrt{x_{1}}+\sqrt{x_{2}}+\sqrt{x_{3}}\right)^{2} & , x_{1} x_{2} x_{3}>0 \\ x_{1}+x_{2}+x_{3} & , x_{1} x_{2} x_{3}=0\end{cases}
$$

is discontinuous. Its continuous extension is $F(\boldsymbol{x})=\left(\sqrt{x_{1}}+\sqrt{x_{2}}+\sqrt{x_{3}}\right)^{2}$. On each coordinate subspace, the function $f$ is obtained from $F$ by reducing all values keeping the function concave. At the subspace $\left\{\boldsymbol{x}=\left(x_{1}, x_{2}, 0\right)\right\}$, the function $F=\left(\sqrt{x_{1}}+\sqrt{x_{2}}\right)^{2}$ is replaced by the smaller concave function $f=x_{1}+x_{2}$, and the same with the other coordinate subspaces. 


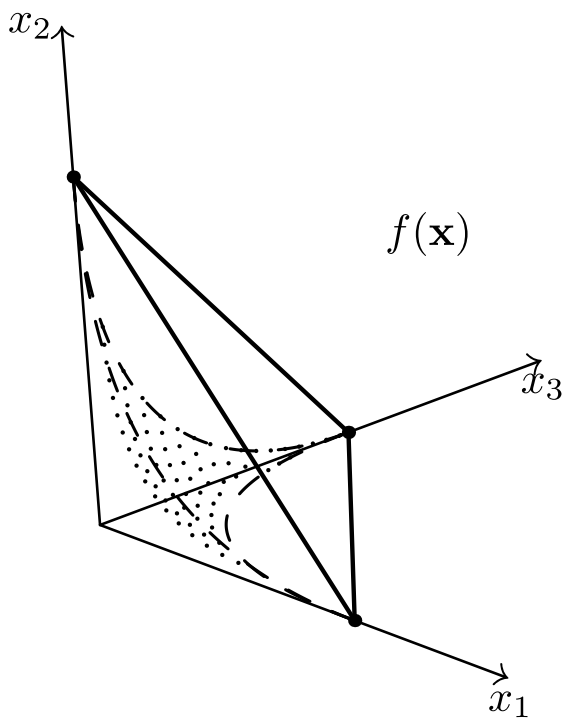

a)

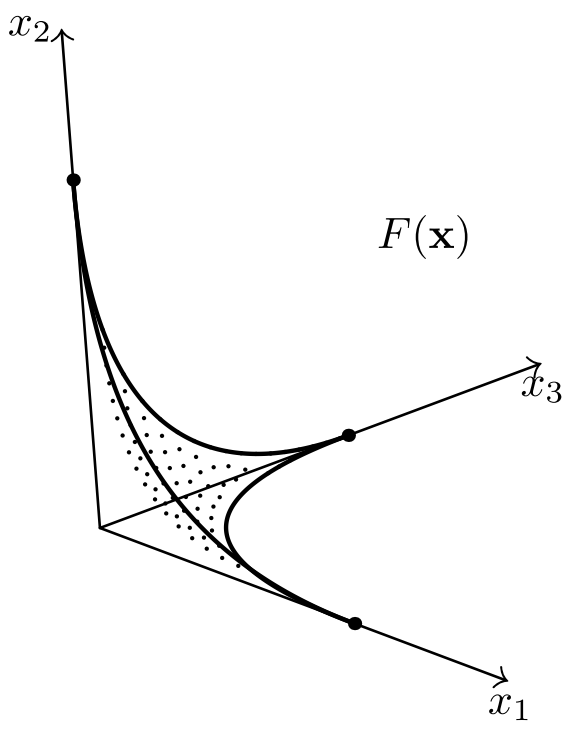

b)

Figure 3: The unit antiball of $f$ (left) and of its continuous extension $F$ (right) from Example 2 .

Remark 3 All antinorms on polyhedral cones admit continuous extensions defined by formula (1). It follows from Proposition 1 that any antinorm on a polyhedral cone has a continuous extension defined by (1). For non-polyhedral cones this may not be true. Indeed, we can consider a Lorentz cone $K \subset \mathbb{R}^{3}$, define the antinorm $f$ on its cross-section disc as in Example 1 and extend it by homegenity onto the whole $K$. Then $f$ does not have a continuous extension. By a similar argument it can be shown that on every non-polyhedral cone, ther eexists an antinorm without continuous extension.

In the next section we study duality of antinorms and prove that an antinorm and its continuous extension have the same dual. This will allow us to replace all antinorms by their extensions, after which we will be able to focus our analysis to continuous antinorms only.

\section{Duality of antinorms}

The dual antinorms on arbitrary cones $K$ were first introduced in [25] and then in a more general form in [15]. The duality theory for antinorms is very similar to convex duality, with the natural replacement of maximum to mininum in the definition of dual functions. In particular, analogues of Young's inequality and of the reflexivity for duality of antinorms are true for this case. On the other hand, there are some differences compared with duality of norms. For example, the double dual coincides not with the original antinorm but with its continuous extension. Another difference is rather surprising: the correspondence between an antinorm and its dual can be discontinuous (even in the class of continuous antinorms). 
Let us remember that the duality of norms is defined in a standard way: if $g(\boldsymbol{x})=\|\boldsymbol{x}\|$ is a norm in $\mathbb{R}^{d}$, then $g^{*}(\boldsymbol{p})=\max _{\boldsymbol{x} \in \mathbb{R}^{d}, \boldsymbol{x} \neq 0} \frac{(\boldsymbol{p}, \boldsymbol{x})}{g(\boldsymbol{x})}$. The unit ball $Q^{*}$ of the dual norm $g^{*}$ is a polar to the unit ball $Q$ of $g$, i.e., $Q^{*}=\left\{\boldsymbol{p} \in \mathbb{R}^{d} \mid(\boldsymbol{p}, \boldsymbol{x}) \leq 1, \boldsymbol{x} \in Q\right\}$.

The duals and polars for antinorms are defined in the same way, replacing maxima by minima and inverting all inequalities. Let $f$ be an antinorm in $\mathbb{R}_{+}^{d}$. Then its dual is

$$
f^{*}(\boldsymbol{p})=\inf _{\boldsymbol{x} \in \mathbb{R}_{+}^{d}, f(\boldsymbol{x}) \neq 0} \frac{(\boldsymbol{p}, \boldsymbol{x})}{f(\boldsymbol{x})}, \quad \boldsymbol{p} \in \mathbb{R}_{+}^{d} .
$$

This definition can be modified as follows. If $f(\boldsymbol{x})=(\boldsymbol{p}, \boldsymbol{x})=0$ (this can occur only on the boundary of the cone), then $\frac{(\boldsymbol{p}, \boldsymbol{x})}{f(\boldsymbol{x})}$ denotes the value $\liminf _{\boldsymbol{y} \rightarrow \boldsymbol{x}} \frac{(\boldsymbol{p}, \boldsymbol{y})}{f(\boldsymbol{y})}$. Being defined this way, the function $\frac{(\boldsymbol{p}, \boldsymbol{x})}{f(\boldsymbol{x})}$ is lower semicontinuous on $\mathbb{R}_{+}^{d}$, and hence possesses a point of absolute minimum. This allows us to write

$$
f^{*}(\boldsymbol{p})=\min _{\boldsymbol{x} \in \mathbb{R}_{+}^{d}} \frac{(\boldsymbol{p}, \boldsymbol{x})}{f(\boldsymbol{x})}, \quad \boldsymbol{p} \in \mathbb{R}_{+}^{d} .
$$

Clearly, the dual function $f^{*}(\boldsymbol{p})$ is also an antinorm. The next observation is less trivial: an antinorm and its continuous extension have the same dual.

Proposition 2 If $f$ is an antinorm and $F$ is its continuous extension, then $f^{*}=F^{*}$.

Proof. Since $F=f$ on the interior of $\mathbb{R}_{+}^{d}$ and $F \geq f$ on the boundary, we have

$$
\min _{\boldsymbol{x} \geq 0} \frac{(\boldsymbol{p}, \boldsymbol{x})}{F(\boldsymbol{x})} \leq \min _{\boldsymbol{x} \geq 0} \frac{(\boldsymbol{p}, \boldsymbol{x})}{f(\boldsymbol{x})} \leq \min _{\boldsymbol{x}>0} \frac{(\boldsymbol{p}, \boldsymbol{x})}{f(\boldsymbol{x})}=\min _{\boldsymbol{x}>0} \frac{(\boldsymbol{p}, \boldsymbol{x})}{F(\boldsymbol{x})} .
$$

On the other hand, the continuity of $F$ implies that $\min _{\boldsymbol{x} \geq 0} \frac{(\boldsymbol{p}, \boldsymbol{x})}{F(\boldsymbol{x})}=\min _{\boldsymbol{x}>0} \frac{(\boldsymbol{p}, \boldsymbol{x})}{F(\boldsymbol{x})}$. Hence, all inequalities in the chain (4) are equalities, therefore $f^{*}(\boldsymbol{p})=F^{*}(\boldsymbol{p})$.

The geometrical meaning of duality is expressed by the antipolar transform $X \rightarrow X^{*}$, where

$$
X^{*}=\left\{\boldsymbol{y} \in \mathbb{R}^{d} \mid(\boldsymbol{y}, \boldsymbol{x}) \geq 1 \text { for all } \boldsymbol{x} \in X\right\}
$$

is the antipolar to the set $X$. We often call it just "polar transform" and will avoid confusion with the standard polar transform. The polar to a point $\boldsymbol{x}$ is the half-space $\left\{\boldsymbol{y} \in \mathbb{R}^{d} \mid(\boldsymbol{x}, \boldsymbol{y}) \geq\right.$ 1 \}, and the polar to the set $X$ is the intersection of those half-spaces over all $\boldsymbol{x} \in X$. It is shown easily that the unit ball of the dual antinorm $f^{*}$ is the polar to the unit ball of $f$.

Example 3 For the antinorm $f(\boldsymbol{x})=\sum_{i=1}^{d} x_{i}$, we have

$$
f^{*}(\boldsymbol{p})=\min _{\boldsymbol{x} \in \mathbb{R}_{+}^{d}, \sum_{i=1}^{d} x_{i}=1} \sum_{i=1}^{d} p_{i} x_{i}=\min \left\{p_{1}, \ldots, p_{d}\right\} .
$$

The unit ball of $f$ is a "conic simplex" $G=\left\{\boldsymbol{x} \in \mathbb{R}_{+}^{d} \mid \sum_{i=1}^{d} x_{i} \geq 1\right\}$. Its polar $G^{*}=\{\boldsymbol{p} \in$ $\left.\mathbb{R}_{+}^{d} \mid p_{i} \geq 1, i=1, \ldots, d\right\}$, which is the unit ball for $f^{*}$, is the positive orthant $\mathbb{R}_{+}^{d}$ shifted to the vector $\boldsymbol{e}=(1, \ldots, 1)^{T}$. 
An analogue of Young's inequality for the Legendre-Fenchel transform follows immediately from the definition of dual antinorms.

Proposition 3 For every antinorm and for every points $\boldsymbol{p}, \boldsymbol{x} \in \mathbb{R}_{+}^{d}$, we have

$$
f^{*}(\boldsymbol{p}) f(\boldsymbol{x}) \geq(\boldsymbol{p}, \boldsymbol{x}) .
$$

The issue of the reflexivity of antinorms was studied in the literature for arbitrary convex cones. It was shown that $f^{* *} \geq f[25]$. Moreover, if an antinorm is continuous, then it is reflexive, i.e., $f^{* *}=f$ [16]. It turns out that the latter result can be generalized to all antinorms, provided the cone is polyhedral. We prove the corresponding theorem for $\mathbb{R}_{+}^{d}$, although it is true for all polyhedral cones.

Theorem 2 For an arbitrary antinorm, we have $f^{* *}=F$, where $F$ is the continuous extension of $F$.

Proof. In view of Proposition 2, it suffices to prove that the duality is reflexive for continuous antinorms. Indeed, in this case, for an arbitrary antinorm $f$, we have $\left(f^{*}\right)^{*}=\left(F^{*}\right)^{*}=F^{* *}=$ $F$. Therefore, we assume that $f$ is continuous and prove that $f^{* *}=f$. To this end we need to show that $f(\boldsymbol{x})=\min _{f^{*}(\boldsymbol{p}) \geq 1}(\boldsymbol{p}, \boldsymbol{x})$. Inequality (6) implies that $f(\boldsymbol{x}) \geq \min _{f^{*}(\boldsymbol{p}) \geq 1}(p, x)$. It remains to establish the inverse inequality. Since $f^{*}(\boldsymbol{p})=\min _{f(\boldsymbol{z}) \geq 1}(\boldsymbol{p}, \boldsymbol{z})$, the inequality $f^{*}(\boldsymbol{p}) \geq 1$ means that $(\boldsymbol{p}, \boldsymbol{z}) \geq 1$ for every $z \in B$, where $B=\left\{\boldsymbol{z} \in \mathbb{R}_{+}^{d} \mid f(\boldsymbol{z}) \geq 1\right\}$. Thus, $(\boldsymbol{p}, \boldsymbol{z}) \geq f(\boldsymbol{z})$ for all $\boldsymbol{z} \in \mathbb{R}_{+}^{d}$. Hence, we need to show that for every $\boldsymbol{x}$ such that $f(\boldsymbol{x}) \leq 1$ there exists $\boldsymbol{p} \in \mathbb{R}_{+}^{d}$ such that $(\boldsymbol{p}, \boldsymbol{x})=1$ and $(\boldsymbol{p}, \boldsymbol{z}) \geq f(\boldsymbol{z})$ for all $\boldsymbol{z}$. By the convex separation theorem, there exists $\boldsymbol{p}$ such that $1=(\boldsymbol{p}, \boldsymbol{x}) \geq \sup _{\boldsymbol{z} \in B}(\boldsymbol{p}, \boldsymbol{z})$, and hence $(\boldsymbol{p}, \boldsymbol{z}) \geq f(\boldsymbol{z})$ for all $\boldsymbol{z}$. It remains to show that $\boldsymbol{p} \in \mathbb{R}_{+}^{d}$. If this is not the case, $\boldsymbol{p}$ is orthogonal to a strictly positive vector $\boldsymbol{a}$. Since $(\boldsymbol{p}, \boldsymbol{z})=(\boldsymbol{p}, \boldsymbol{z}+\lambda \boldsymbol{a}) \geq f(\boldsymbol{z}+\lambda \boldsymbol{a})$ for all $\lambda>0$. Thus, $\lambda^{-1}(\boldsymbol{p}, \boldsymbol{z}) \geq f\left(\lambda^{-1} \boldsymbol{z}+\boldsymbol{a}\right)$, which in the limit as $\lambda \rightarrow+\infty$ gives $0 \geq f(\boldsymbol{a})$. This is impossible since $f(\boldsymbol{a})>0$ at the positive point $\boldsymbol{a}$.

As a corollary, we obtain the result from [16]:

Corollary 2 If an antinorm $f$ is continuous, then $f^{* *}=f$.

Proposition 2 and Theorem 2 allows us to restrict naturally our analysis to the set of continuous antinorms. Thus, in what follows, all antinorms are supposed to be continuous if the converse is not stated.

We see that the duality map $f \mapsto f^{*}$ constitutes a reflexive transform on the set of antinorms. Surprisingly enough, this transform is discontinuous, even on the set of continuous antonorms. This makes one more difference from duality of norms.

Naturally, the distance between antimorms is defined as the maximal difference between them on the unit simplex $\Delta=\left\{\boldsymbol{x} \in \mathbb{R}_{+}^{d} \mid(\boldsymbol{e}, \boldsymbol{x})=1\right\}$, where $\boldsymbol{e}$ is the vector of ones. Thus, $\left\|f_{1}-f_{2}\right\|=\max _{\boldsymbol{x} \in \Delta}\left|f_{1}(\boldsymbol{x})-f_{2}(\boldsymbol{x})\right|$.

Theorem 3 The map $f \mapsto f^{*}$ is discontinuous. 
Proof. We present an example in $\mathbb{R}^{2}$, which is easily extended to higher dimensions. Consider the following family of antinorms on $\mathbb{R}_{+}^{2}$ :

$$
f_{\varepsilon}(x, y)=\min \{x, y\}+\varepsilon \sqrt{x y}, \quad(x, y) \in \mathbb{R}_{+}^{2},
$$

where $\varepsilon \in[0,1]$. In particular, $f_{0}(x, y)=\min \{x, y\}$. Let us show that $f_{\varepsilon}^{*}$ does not converge to $f_{0}^{*}$ as $\varepsilon \rightarrow 0$. We have

$$
f_{0}^{*}(p, q)=\min _{(x, y) \in \mathbb{R}_{+}^{2}} \frac{p x+q y}{\min \{x, y\}}=\min _{0 \leq x \leq y} \frac{p x+q y}{x}=p+\min _{0 \leq x \leq y} \frac{q y}{x}=p+q .
$$

Thus,

$$
f_{0}^{*}(p, q)=p+q .
$$

Now compute $f_{\varepsilon}^{*}(p, q)$ with $\varepsilon>0$. We do it only for points $(p, q)$ satisfying the assumption

$$
q \leq \frac{\varepsilon^{2}}{8} p
$$

We have

$$
f_{\varepsilon}^{*}(p, q)=\min _{(x, y) \in \mathbb{R}_{+}^{2}} \frac{p x+q y}{\min \{x, y\}+\varepsilon \sqrt{x y}}=\min _{0 \leq x \leq y} \frac{p x+q y}{x+\varepsilon \sqrt{x y}}=\min _{0<x \leq y} \frac{p+q \frac{y}{x}}{1+\varepsilon \sqrt{\frac{y}{x}}} .
$$

We denote $\sqrt{\frac{y}{x}}=t$ and rewrite as follows:

$$
f_{\varepsilon}^{*}(p, q)=\min _{t \geq 1} \frac{p+q t^{2}}{1+\varepsilon t} .
$$

Denote this minimum by $m$. This is the smallest parameter value for which the equation

$$
q t^{2}-m \varepsilon t+p-m=0 .
$$

has a root $t \geq 1$. If $t=1$, then $m=\frac{p+q}{1+\varepsilon}$. If $t>1$, then the discriminant

$$
\varepsilon^{2} m^{2}+4 q m-4 q p=0,
$$

hence

$$
m=\frac{-2 q+\sqrt{4 q^{2}+4 \varepsilon^{2} p q}}{\varepsilon^{2}}=\frac{4 p q}{2 q+\sqrt{4 q^{2}+4 \varepsilon^{2} p q}}=\frac{2 p q}{q+\sqrt{q^{2}+\varepsilon^{2} p q}} .
$$

By (8), the last expression does not exceed $\frac{p+q}{1+\varepsilon}$. Thus,

$$
f_{\varepsilon}^{*}(p, q)=\frac{2 p q}{q+\sqrt{q^{2}+\varepsilon^{2} p q}}, \quad q \leq \frac{\varepsilon^{2}}{8} p
$$


This implies that

$$
f_{\varepsilon}^{*}(p, q) \leq \frac{2}{\varepsilon} \sqrt{q p}
$$

Therefore, $f_{\varepsilon}^{*}(1,0)=0$ for all $\varepsilon$, while $f_{0}^{*}(1,0)=1+0=1$ as follows from (7). This completes the proof.

In the next section we characterize self-dualily. We will see that this issue for antinorms is more interesting and challenging than for norms.

\section{Self-dual antinorms}

An antinorm is called self-dual if $f^{*}=f$. Before studying self-dual antinorms, let us remember that the situation with self-dual norms is very simple. Self-duality is an exclusive property of the Euclidean norm.

Fact. The unique self-dual norm in $\mathbb{R}^{d}$ is the Euclidean norm.

For convenience of the reader, we include the proof of this classical fact.

Proof. Assume there exists a point $\boldsymbol{x} \in \mathbb{R}^{d}$ whose self-dual norm $f(\boldsymbol{x})$ is strictly smaller than its Euclidean norm $|\boldsymbol{x}|$. Then $f^{*}(\boldsymbol{x})=\max _{\boldsymbol{z} \in \mathbb{R}^{d} \backslash\{0\}} \frac{(\boldsymbol{x}, \boldsymbol{z})}{f(\boldsymbol{z})} \geq \frac{(\boldsymbol{x}, \boldsymbol{x})}{f(\boldsymbol{x})}>\frac{(\boldsymbol{x}, \boldsymbol{x})}{\|\boldsymbol{x}\|}=|\boldsymbol{x}|$. Hence, $f(\boldsymbol{x})=f^{*}(\boldsymbol{x})>|\boldsymbol{x}|$, which contradicts to the assumption. Thus, $f(\boldsymbol{x}) \geq|\boldsymbol{x}|$ for all $\boldsymbol{x}$. Assume $f(\boldsymbol{x})>|\boldsymbol{x}|$ for some $\boldsymbol{x}$. In this case $f^{*}(\boldsymbol{x})>|\boldsymbol{x}|$, and hence there exists $\boldsymbol{z} \in \mathbb{R}_{+}^{d}$ such that $\frac{(\boldsymbol{x}, \boldsymbol{z})}{f(\boldsymbol{z})}>|\boldsymbol{x}|$. Since $(\boldsymbol{x}, \boldsymbol{z}) \leq\|\boldsymbol{x}\| \cdot\|\boldsymbol{z}\|$, it follows that $|\boldsymbol{x}| \cdot|\boldsymbol{z}|>|\boldsymbol{x}| \cdot f(\boldsymbol{z})$, therefore $|\boldsymbol{z}|>f(\boldsymbol{z})$, which is a contradiction.

Remark 4 The statement above means that the unit Euclidean ball is the only autopolar set in $\mathbb{R}^{d}$. One may wonder about autopolar triangles or autopolar simplices, which are well-known. All of them are actually not autopolar: their "autopolarity" means that the set of vertices is polar to the set of sides (or, in $\mathbb{R}^{d}$, the set of hyperfaces).

In contrast, in every dimension $d \geq 2$, there are infinitely many different self-dual antinorms, or, which is the same, infinitely many autopolar conic bodies. One of families of such antinorms is provided by the following assertion.

Proposition 4 Let $\left\{p_{i}\right\}_{i=1}^{d}$ be a collection of non-negative numbers such that $\sum_{i=1}^{d} p_{i}=1$. Then the function $f(\boldsymbol{x})=\sqrt{d} \prod_{i=1}^{d} x_{i}^{p_{i}}$ is a self-dual antinorm.

Proof. The concavity is well-known. We find $f^{*}(x)$ from the problem

$$
\left\{\begin{array}{ccc}
-\sqrt{d} \prod_{i=1}^{d} z_{i}^{p_{i}} & \rightarrow & \min \\
\sum_{i=1}^{d} x_{i} z_{i} & = & 1
\end{array}\right.
$$


This is a convex problem, so its minimum is computed by the Karush-Kuhn-Tucker theorem: there exists $\lambda \geq 0$ such that $\mathcal{L}_{\boldsymbol{z}}(\boldsymbol{z}, \lambda)=0$, where $\mathcal{L}=-\sqrt{d} \prod_{i=1}^{d} z_{i}^{p_{i}}+\lambda\left(\sum_{i=1}^{d} x_{i} z_{i}-1\right)$. For each $i$, we have $\mathcal{L}_{z_{i}}=-\frac{p_{i}}{z_{i}} \sqrt{d} \prod_{i=1}^{d} z_{i}^{p_{i}}+\lambda x_{i}=0$. Therefore $x_{i} z_{i}=c$ for all $i$. Substituting to the constraint $\sum_{i=1}^{d} x_{i} z_{i}=1$, we get $c=\frac{1}{d}$, hence the point of minimum is $z_{i}=1 /\left(d x_{i}\right)$. Consequently,

$$
f^{*}(\boldsymbol{x})=\frac{(\boldsymbol{x}, \boldsymbol{z})}{\sqrt{d} \prod_{i=1}^{d} z_{i}^{p_{i}}}=\frac{1}{\sqrt{d} \prod_{i=1}^{d}\left(1 / d x_{i}\right)^{p_{i}}}=\sqrt{d} \prod_{i=1}^{d} x_{i}^{p_{i}}=f(\boldsymbol{x}) .
$$

This completes the proof.

\subsection{Basic properties of self-dual antinorms}

The geometrical meaning of self-duality is that the unit ball $G=\left\{\boldsymbol{x} \in \mathbb{R}_{+}^{d} \mid f(\boldsymbol{x}) \geq 1\right\}$ is autopolar: $G^{*}=G$, where the antipolar $G^{*}$ is defined in (5). According to that definition, the polar to a point $\boldsymbol{x}$ is the half-space $\left\{\boldsymbol{y} \in \mathbb{R}^{d} \mid(\boldsymbol{x}, \boldsymbol{y}) \geq 1\right\}$. The polar hyperplane $p(\boldsymbol{x})$ is defined as the boundary of that subspace: $p(\boldsymbol{x})=\left\{\boldsymbol{y} \in \mathbb{R}^{d} \mid(\boldsymbol{x}, \boldsymbol{y})=1\right\}$. The point $\boldsymbol{x}$ is called the pole of this hyperplane. We usually call the polar hyperplane simply polar, when it is clear that we mean the plane but not the half-space. Let $\mathcal{S}=\left\{\boldsymbol{x} \in \mathbb{R}^{d} \mid f(\boldsymbol{x})=1\right\}$ be the antisphere of $f$. The antisphere is autopolar if the set of its hyperplanes of support coincides with $\{p(\boldsymbol{x}) \mid \boldsymbol{x} \in \mathcal{S}\}$. This is equivalent to the autopolarity of the ball $G$. The proof of the following fact is simple and we omit it.

Proposition 5 An antinorm is self-dual if and only if its antisphere is autopolar.

Another property of self-dual antinorms is that all of them are smaller than the Euclidean norm in all but one direction, i.e., for every antinorm, there exists a unique direction where it is equal to the Euclidean norm.

Proposition 6 For every self-dual antinorm $f$, we have $f(\boldsymbol{x}) \leq|\boldsymbol{x}|, \boldsymbol{x} \in \mathbb{R}_{+}^{d}$, and there is a unique up to normalization vector $\boldsymbol{a} \in \mathbb{R}_{+}^{d}, \boldsymbol{a} \neq 0$, such that $f(\boldsymbol{a})=|\boldsymbol{a}|$.

Proof. On the unit antisphere $\mathcal{S}$, we chose the point $\boldsymbol{a}$ closest to the origin. We have $1=f(\boldsymbol{a})=f^{*}(\boldsymbol{a})=\min _{f(\boldsymbol{x})>0} \frac{(\boldsymbol{x}, \boldsymbol{a})}{f(\boldsymbol{x})} \leq \frac{(\boldsymbol{a}, \boldsymbol{a})}{f(\boldsymbol{a})}=(\boldsymbol{a}, \boldsymbol{a})$. Hence $|\boldsymbol{a}| \geq 1$. On the other hand, if $|\boldsymbol{a}|>1$, then the polar of the point $P$ strictly separates the whole antisphere $\mathcal{S}$ from the origin. This contradicts to the self-duality. Hence, $|\boldsymbol{a}|=1$ and $f(\boldsymbol{x}) \leq(\boldsymbol{a}, \boldsymbol{x})$. Such a point $\boldsymbol{a} \in \mathcal{S}$ is unique. Indeed, if there are two of them $\boldsymbol{a}_{1}, \boldsymbol{a}_{2}$, then for the point $\boldsymbol{c}=\frac{1}{2}\left(\boldsymbol{a}_{1}+\boldsymbol{a}_{2}\right)$, we have $|\boldsymbol{c}|<1$ and $f(\boldsymbol{c}) \geq 1$. Hence, the point $\frac{1}{f(\boldsymbol{c})} \boldsymbol{c}$ belongs to $\mathcal{S}$ and has the norm less than one, which is impossible.

Thus, the vector $\boldsymbol{a}$ in Proposition 6 is the closest point of the antispere $\mathcal{S}$ to the origin and $|\boldsymbol{a}|=1$. 
Corollary 3 For every self-dual antinorm, its antisphere has a unique point of intersection with the Euclidean unit sphere. This is the point a form Proposition 6.

\subsection{Classification of self-dual antinorms}

Once there are infinitely many self-dual antinorms in $\mathbb{R}^{d}$, the question arises about their possible classification. For the plane $(d=2)$ this problems admits a complete solution. First, we consider the following

\section{Construction 1.}

1) take arbitrary $\boldsymbol{a} \in \mathbb{R}_{+}^{2},|\boldsymbol{a}|=1$, and draw a ray $\left\{t \boldsymbol{a} \mid t \in \mathbb{R}_{+}\right\}$, which splits the positive orthant $\mathbb{R}_{+}^{2}$ into two angles $K_{1}$ and $K_{2}$ (one of them may be degenerate);

2) choose an arbitrary antinorm $f_{1}$ on $K_{1}$ such that $f_{1}(\boldsymbol{a})=1$ and $f_{1}(\boldsymbol{x}) \leq(\boldsymbol{a}, \boldsymbol{x}), \boldsymbol{x} \in K_{1}$;

3) define the function $f_{2}$ on $K_{2}$ as a dual to $f_{1}: f_{2}\left(\boldsymbol{x}_{2}\right)=\inf _{\boldsymbol{x}_{1} \in K_{1}} \frac{\left(\boldsymbol{x}_{1}, \boldsymbol{x}_{2}\right)}{f_{1}\left(\boldsymbol{x}_{1}\right)}$. Then define $f: \mathbb{R}_{+}^{2} \rightarrow \mathbb{R}_{+}$as follows:

$$
f(\boldsymbol{x})=\left\{\begin{array}{l}
f_{1}(\boldsymbol{x}), \boldsymbol{x} \in K_{1} \\
f_{2}(\boldsymbol{x}), \boldsymbol{x} \in K_{2}
\end{array}\right.
$$

Theorem 4 All self-dual antinorms in $\mathbb{R}_{+}^{2}$ are precisely those defined by formula (11) with functions $f_{1}, f_{2}$ obtained by Construction 1.

Before giving a proof we make one comment.

Remark 5 The self-duality of the antinorm $f$ defined in (11) is not quite obvious, because $f_{2}$ is the dual to $f_{1}$ only on the cone $K_{1}$, but not in the entire $\mathbb{R}_{+}^{2}$. To prove that actually $f_{2}^{*}=f_{1}$ and vice versa one needs to show that for every $\boldsymbol{x}_{2} \in K_{2}$, the minimum $\min _{\boldsymbol{x}_{1} \in \mathbb{R}_{+}^{2}} \frac{\left(\boldsymbol{x}_{1}, \boldsymbol{x}_{2}\right)}{f_{1}\left(\boldsymbol{x}_{1}\right)}$ is attained for $\boldsymbol{x}_{1} \in K_{1}$.

Proof of Theorem 4. Let $\mathcal{S}_{1}=\left\{\boldsymbol{x} \in K_{1} \mid f_{1}(\boldsymbol{x})=1\right\}$ be the antisphere of the antinorm $f_{1}$ in $K_{1}$. Let $O P=\boldsymbol{a}$. By the assumption, $P \in \mathcal{S}_{1}$. For an arbitrary point $A \in \mathcal{S}_{1}$, denote by $B$ the intersection of the segment $O A$ with the line orthogonal to $O P$ passing through $P$, and by $A^{\prime}, B^{\prime}$ points inverse to $A, B$ respectively (Fig. 4). Since $|O A| \geq|O B|$ we have $\left|O A^{\prime}\right| \leq\left|O B^{\prime}\right|$. Since in the right triangle $O P B$, the square of the leg $O P$ is equal to its projection to the hypotenuse $O B$ multiplied by $O B$, we see that the length of the projection is $|O P|^{2} /|O B|=1 /|O B|=\left|O B^{\prime}\right|$. Hence $P B^{\prime}$ is the polar $p(B)$. Therefore, the polar $p(A)$, which is parallel to $p(B)$ and passes through $A^{\prime}$, is closer to $O$ than $p(B)$. Hence, the line $p(B)$ separates $p(A)$ from $\mathcal{S}_{1}$. Thus, the polar $p(A)$ of an arbitrary point $A \in \mathcal{S}_{1}$ does not intersect the line $\mathcal{S}_{1}$ expect possibly at $P$. Hence, the whole polar image of $\mathcal{S}_{1}$ is located in $K_{2}$ and therefore coincides with $\mathcal{S}_{2}$. Then, by the reflexivity of the polar transform, the polar image of $\mathcal{S}_{1}$ is located in $K_{2}$ and therefore coincides with $\mathcal{S}_{2}$. Thus, the polar transform interchanges $\mathcal{S}_{1}$ and $\mathcal{S}_{2}$, and $\mathcal{S}$ is self-polar. 


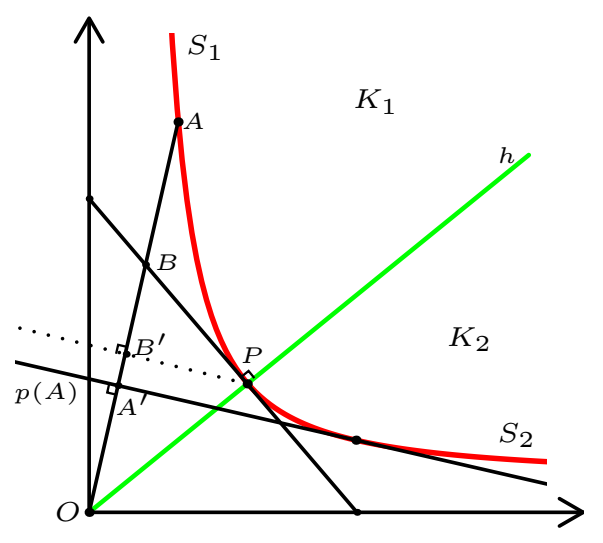

Figure 4: Proof of Theorem 4

Conversely, let $f$ be a self-dual antinorm and $\mathcal{S}$ be its antisphere. By Proposition 6, $\mathcal{S}$ has a unique closest point $\boldsymbol{a}$ to the origin and $|\boldsymbol{a}|=1$. Denote $P=\boldsymbol{a}$. Let $h$ be the line orthogonal to $O P$ and passing through $P$. The whole antisphere $\mathcal{S}$ lies on the opposite side from the origin about $h$. Then the ray $\left\{t \boldsymbol{a}, t \in \mathbb{R}_{+}\right\}$splits the orthant $\mathbb{R}_{+}^{2}$ into two angles $K_{1}$ and $K_{2}$. Then the function $f_{1}=\left.f\right|_{K_{1}}$ generates the function $f$ by the procedure 1-3 in the theorem.

Remark 6 Geometrically, Theorem 4 gives the following characterization of self-dual antinorms on the plane. We split the positive orthant $\mathbb{R}_{+}^{2}$ by some ray $\{t \boldsymbol{\alpha} \mid t \geq 0\}$ into two angles $K_{1}, K_{2}$ according to item 1 ) of Construction 1 and then take an arbitrary antinorm $f_{1}$ in $K_{1}$ whose antisphere $\mathcal{S}_{1}$ lies above the perpendicular to that ray through $\boldsymbol{a}$. Then $\mathcal{S}_{2}$ is a polar of $\mathcal{S}_{1}$. Concatenation of $\mathcal{S}_{1}$ and of $\mathcal{S}_{2}$ gives the antisphere of $f$. Thus, the curve $\mathcal{S}_{1}$ defines the antinorm $f$.

Example 4 Let $\boldsymbol{a}=\frac{\sqrt{2}}{2}(1,1)$; then the ray generated by $\boldsymbol{a}$ is the bisector of the coordinate angle $X O Y$. On this bisector we take a point $(R, R)$, where $R=1+\sqrt{2}$, and draw a circle of radius $R$ centered at this point. This circle is tangent to the axes and passes through $\boldsymbol{a}$. We take as $\mathcal{S}_{1}$ the arc of this circle connecting the point $\boldsymbol{a}$ with the point of tangency with the axis $O Y$. Then the polar $\mathcal{S}_{2}$ of this arc is the piece of hyperbola $y=\frac{1}{x-a}+b$, starting at $\boldsymbol{a}$ and going along the axis $O X$ to $+\infty$, where $a=\frac{3 \sqrt{2}}{2}-2, b=\sqrt{2}-1$. It asymptotically tends to the line $y=\sqrt{2}-1$. Then the union of $\mathcal{S}_{1}$ and $\mathcal{S}_{2}$ is the autopolar antisphere.

If $\mathcal{S}_{1}$ is a broken line, then $\mathcal{S}_{2}$ is a broken line as well and $f$ is a piecewise linear function. Hence, there are infinitely many autopolar conic polygons in $\mathbb{R}_{+}^{2}$ (in the sense of "antipolar") and we have classified them all. In the next subsection we give their explicit description. 


\subsection{Autopolar conic polygons and polyhedra}

The class of piecewise-linear antinorms is especially important, since such antinorms are easily described. A piecewise-linear antinorm has the form $f(\boldsymbol{x})=\min _{j=1, \ldots, n}\left(\boldsymbol{a}_{j}, \boldsymbol{x}\right)$, where $\boldsymbol{a}_{j}$ are nonnegative vectors. The unit ball of a piecewise-linear antinorm is a conic polyhedron (see Definition 2). A conic polygon is a two-dimensional conic polyhedron .

Theorem 4 allows us to provide an explicit construction of all autopolar conic polygons. Construction 2. We build a broken line $A_{-k} \ldots A_{-1} A_{0} A_{1} \ldots A_{k-1}$ in $\mathbb{R}_{+}^{2}$ as follows.

Take an arbitrary point $A_{0}$ such that $O A_{0}=1$. Draw a line through $A_{0}$ orthogonal to $O A_{0}$ and take an arbitrary point $A_{-1}$ on it. Drop a perpendicular from $A_{0}$ to $O A_{-1}$ and take an arbitrary point $A_{1}$ on its extension through $A_{0}$. The following is by induction. If the point $A_{j}$ is constructed (assume $j>0$ ), then we drop a perpendicular from $A_{-j}$ to $O A_{j}$ and take an arbitrary point $A_{-j-1}$ on its extension through $A_{-j}$. If $j<0$, then we drop a perpendicular from $A_{-j-1}$ to $O A_{j}$ and take an arbitrary point $A_{-j}$ on its extension through $A_{-j-1}($ Fig $[5)$.

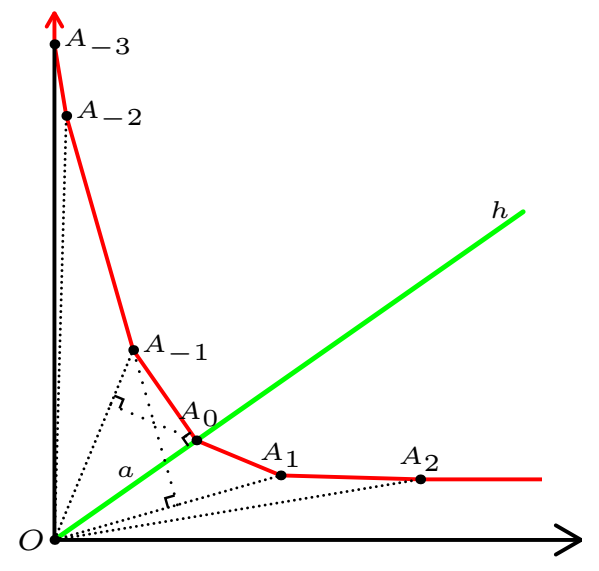

Figure 5: Construction of an autopolar polygon for $k=3$

In the last iteration we choose the point $A_{-k}$ on the coordinate axis $O Y$. Draw a ray from $A_{-k}$ along the axis $O Y$ and a ray from $A_{k-1}$ parallel to the axis $O X$; call those rays $A_{-k} Y$ and $A_{k-1} X$ respectively. Then $Y A_{-k} \ldots A_{-1} A_{0} A_{1} \ldots A_{k-1} X$ is the desired autopolar conic polygon. Invoking Theorem 4 we conclude

Corollary 4 Every self-dual conic polygon is obtained by Construction 2.

For each $k$, that conic polygon has $2 k$ vertices (the vertex $A_{-k}$ is on the axis, all others are strictly inside $\mathbb{R}_{+}^{2}$ ) and $2 k+1$ sides (the sides $A_{-k} Y$ and $A_{k-1} Y$ are rays, all others are segments). The simplest cases are described in the following examples:

Example $5(k=0)$. This case is formally out of our construction. Here $A_{0}=(1,0)$ and the side $A_{0} A_{-1}$ becomes the vertical ray from $A_{0}$. Thus, the polygon has one vertex $A_{0}$ and two sides that are rays from $A_{0}$ parallel to the coordinate axes. The antinorm is $f(x, y)=x$. 
Example $6(k=1)$. Here $A_{0}$ is an interior point of $\mathbb{R}_{+}^{2}$ and $A_{-1}$ is a point on the axis $O Y$ such that $\angle A_{-1} A_{0} O=90^{\circ}$. Then the self-dual polygon is bounded by the rays $A_{-1} Y, A_{0} X$ and by the segment $A_{-1} A_{0}$. If $A_{0}=(a, b)$, then $f(x, y)=\min \left\{a x+b y, \frac{y}{b}\right\}$.

Characterisation of self-dual antinorms, in particular, polyhedral antinorms, in higher dimensions, are left as open problems and discussed in Section 5.

\subsection{Symmetric antinorms}

An antinorm is called symmetric if it is invariant with respect to every permutation of coordinates. There is a variety of symmetric antinorms. Choosing a parameter $p \in[-\infty, 1]$, we define the $L_{p}$-symmetrization of an arbitrary antinorm $f$ as follows:

$$
f^{[p]}\left(x_{1}, \ldots, x_{d}\right)=\left[\frac{1}{d !} \sum_{\sigma} f^{p}\left(x_{\sigma(1)}, \ldots, x_{\sigma(d)}\right)\right]^{1 / p},
$$

with natural modifications for $p=0$ (where the $L_{p}$-mean becomes the geometrical mean) and for $p=-\infty$ (the $L_{p}$-mean becomes the minimum). The sum is computed over all permutations of the set $\{1, \ldots, d\}$. Then $f^{[p]}$ is a symmetric antinorm.

Are there symmetric self-dual antinorms? Yes, for example, $f(\boldsymbol{x})=\sqrt{d}\left(x_{1} \cdots x_{d}\right)^{1 / d}$. This is the antinorm from Proposition 4 with all $p_{i}$ equal to $1 / d$. What about others, do they exist? In case of negative answer we come to the following situation: although there are many self-dual antinorms, in the class of symmetric antinorms it is unique. We are going to see that at least for $d=2$ this is true.

A bivariate antinorm $f(x, y)$ on $\mathbb{R}_{+}^{2}$ is symmetric if $f(x, y)=f(y, x)$. We prove that in the rich variety of self-dual antinorms on $\mathbb{R}_{+}^{2}$ (see Theorem 4 ), there is only one symmetric antinorm.

Theorem 5 The unique symmetric self-dual antinorm on $\mathbb{R}_{+}^{2}$ is $f(x, y)=\sqrt{2 x y}$.

First, we realize the proof under the assumption that $f$ is smooth, i.e., is differentiable at each interior point of $\mathbb{R}_{+}^{2}$. Geometrically this means that there is a unique line of support to the antiball $G=\left\{\boldsymbol{x} \in \mathbb{R}_{+}^{2} \mid f(\boldsymbol{x}) \geq 1\right\}$ at every point of its boundary $\mathcal{S}=\partial B$. In this case we call the unique line of support tangent line to $\mathcal{S}$. Then we extend the proof to the nonsmooth case. We use two simple lemmas.

Lemma 2 If a monotone function $\varphi: \mathbb{R}_{+} \rightarrow \mathbb{R}_{+}$is such that $\varphi(0)=0$ and $\varphi(\varphi(u)) \equiv u$, then $\varphi(u) \equiv u$.

Proof. From the assumption it follows that $\varphi$ is non-decreasing. If $\varphi(u)>u$ for some $u$, then taking $\varphi$ of both sides of the equation we obtain $\varphi(\varphi(u)) \geq \varphi(u)>u$, hence $\varphi(\varphi(u))>u$, which is impossible. Similarly, if $\varphi(u)<u$, then $\varphi(\varphi(u))<u$. The contradiction proves that $\varphi(u)=u$ for all $u \geq 0$.

Now we are able to prove the following generalization of Lemma 2 
Lemma 3 Let a set $\Omega \subset \mathbb{R}_{+}$consist of disjoint nonempty half-open intervals $\left(a_{i}, b_{i}\right], i \in \mathcal{I}$, where the index set $\mathcal{I}$ is either finite or coincides with $\mathbb{N}$. For each $u \in \mathbb{R}_{+}$, we define the number $\tilde{u}$ as follows:

$$
\tilde{u}= \begin{cases}u, & u \notin \Omega, \\ a_{i}, & u \in\left(a_{i}, b_{i}\right], i \in \mathcal{I} .\end{cases}
$$

Then, if a monotone function $\varphi: \mathbb{R}_{+} \rightarrow \mathbb{R}_{+}$which is constant on each interval $\left(a_{i}, b_{i}\right], i \in \mathcal{I}$, and $\varphi(0)=0$, satisfies the inequality $\varphi(\varphi(u))=\tilde{u}$, then $\varphi(u)=\tilde{u}$.

Proof. From the assumptions it follows that $\varphi$ is non-decreasing. If $\varphi(u)>u$ for some $u$, then $\varphi(\varphi(u)) \geq \varphi(u)>u$, which is impossible, because (12) implies that $\varphi(\varphi(u)) \leq u$ for all $u$. Consequently, $\varphi(u) \leq u$ for all $u$. If $u \notin \Omega$, then $\varphi(\varphi(u))=u$ and we conclude as in the proof of Lemma 2 that $\varphi(u)=u$. If $u \in\left(a_{i}, b_{i}\right]$, then $\varphi(u) \leq u \leq b_{i}$. If $\varphi(u)<a_{i}$, then $\varphi(\varphi(u)) \leq \varphi\left(a_{i}\right) \leq \varphi(u)<a_{i}$, which is impossible due to [12). Therefore, $\varphi(u) \in\left(a_{i}, b_{i}\right]$. Since $\varphi$ is constant on the interval $\left(a_{i}, b_{i}\right]$ and both $u$ and $\varphi(u)$ belong to it, we see that $\varphi(u)=\varphi(\varphi(u))=a_{i}$, which completes the proof.

Proof of Theorem 5. Let $f$ be a self-dual antinorm with unit antisphere $\mathcal{S}$. By Proposition 6, $|\boldsymbol{x}| \geq 2$ for all $\boldsymbol{x} \in \mathcal{S}$, and there exists a unique $\boldsymbol{a} \in \mathcal{S}$ such that $|\boldsymbol{a}|=1$. If $f$ is symmetric, then $\boldsymbol{a}$ is symmetric to itself, and therefore $\boldsymbol{a}=\left(\frac{1}{\sqrt{2}}, \frac{1}{\sqrt{2}}\right)$ (Fig. 6a).

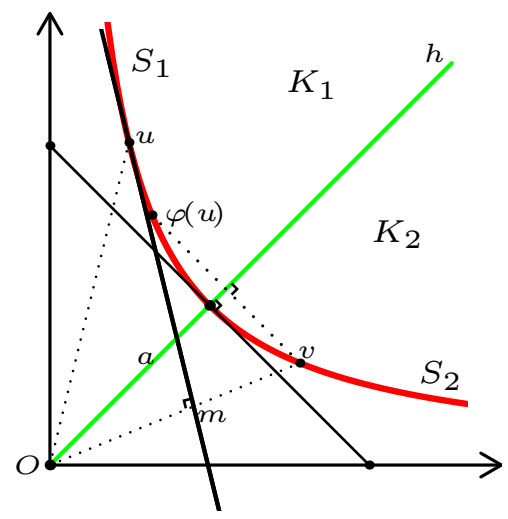

a)

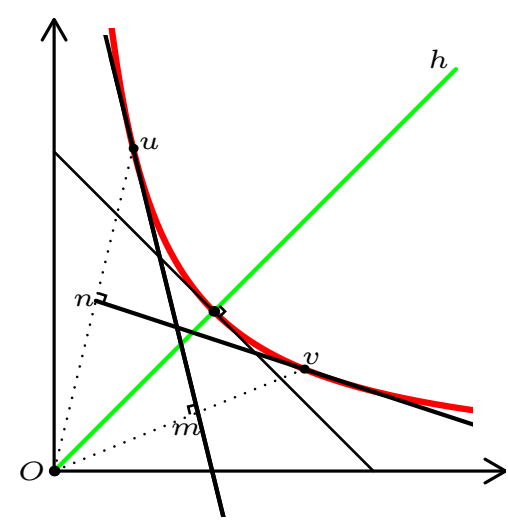

b)

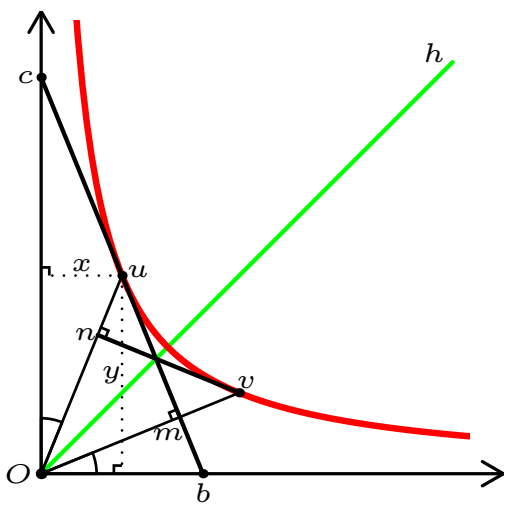

c)

Figure 6: Proof of Theorem 5. The smooth case.

The smooth case. Define a map $\varphi: \mathcal{S} \rightarrow \mathcal{S}$ as follows: for every $\boldsymbol{u} \in \mathcal{S}$, the point $\varphi(\boldsymbol{u})$ is symmetric to the pole of the tangent line to $\mathcal{S}$ at the point $\boldsymbol{u}$. Denote this pole by $\boldsymbol{v}$ (Fig. 6a). Clearly, $\boldsymbol{v} \in \mathcal{S}$. The self-duality implies that $\boldsymbol{u}$ is a pole to the tangent line to $\mathcal{S}$ at the point $\boldsymbol{v}$. Hence, $\varphi(\boldsymbol{v})$ is a point symmetric to $\boldsymbol{u}$.

Let $\boldsymbol{u}^{\prime}, \boldsymbol{v}^{\prime}$ be points symmetric to $\boldsymbol{u}$ and $\boldsymbol{v}$ respectively (fig. 6 b). We have $\varphi(\boldsymbol{v})=\boldsymbol{u}^{\prime}$ and due to the symmetry, $\varphi\left(\boldsymbol{v}^{\prime}\right)=\boldsymbol{u}$. But $\boldsymbol{v}^{\prime}=\varphi(\boldsymbol{u})$. Therefore, $\varphi(\varphi(\boldsymbol{u}))=\boldsymbol{u}$. The point $\boldsymbol{a}$ splits the curve $\mathcal{S}$ into two parts $\mathcal{S}_{1}$ and $\mathcal{S}_{2}$. Consider a natural parametrization of the curve $\mathcal{S}_{1}$ by the length of the arc from a point to $\boldsymbol{a}$. It defines a homeomorphim of $\mathcal{S}_{1}$ and $\mathbb{R}_{+}$. Let a point $\boldsymbol{u}$ be associated to a parameter $u$ and we keep the notation $\varphi$ for the 
corresponding map on the half-line. We have $\varphi(\varphi(u))=u$ for all $u \in \mathbb{R}_{+}$and by Lemma 2 , $\varphi(u)=u$. Therefore, $\varphi(\boldsymbol{u})=\boldsymbol{u}$, i.e., every point $\boldsymbol{u}$ is symmetric to the pole $\boldsymbol{v}$ of the tangent line passing through $\boldsymbol{u}$.

Denote by $\boldsymbol{m}, \boldsymbol{b}$, and $\boldsymbol{c}$ the points of intersection of that tangent line with the segment $O \boldsymbol{v}$ and with the coordinate axes $O X$ and $O Y$ respectively. We have $O \boldsymbol{m} \cdot O \boldsymbol{v}=1$. Due to the symmetry, $\angle \boldsymbol{b} O \boldsymbol{v}=\angle \boldsymbol{c O u}$. On the other hand, $O \boldsymbol{m}$ is the altitude of the right triangle $\boldsymbol{b} O \boldsymbol{c}$ to the hypotenuse $\boldsymbol{b} \boldsymbol{c}$ (Fig. 6]c). Hence $\angle \boldsymbol{b} O \boldsymbol{v}=\angle O \boldsymbol{c u}$. Thus, $\angle \boldsymbol{u} O \boldsymbol{c}=\angle O \boldsymbol{c u}$ and so $O \boldsymbol{u}$ is the midpoint of the hypotenuse $\boldsymbol{b} \boldsymbol{c}$. Hence the median $O \boldsymbol{u}$ is equal to the half of the hypotenuse $\boldsymbol{b} \boldsymbol{c}$. The area of the triangle $\boldsymbol{b} O \boldsymbol{c}$ is equal to $\frac{1}{2} O \boldsymbol{m} \cdot \boldsymbol{b c}=O \boldsymbol{m} \cdot \mathrm{Ou}=$ $O \boldsymbol{m} \cdot O \boldsymbol{v}=1$. On the other hand, the same area is equal to $\frac{1}{2} O \boldsymbol{b} \cdot O \boldsymbol{c}=\frac{1}{2} 2 x \cdot 2 y=2 x y$, where $x, y$ are the coordinates of the point $\boldsymbol{u}$ (Fig. 6]c). Thus, $2 x y=1$ for every point $\boldsymbol{u} \in \mathcal{S}$. Hence, $f(x, y)=1$ if and only if $2 x y=1$, and by homogeneity $f(x, y)=\sqrt{2 x y}$.

The non-smooth case. Define a map $\varphi: \mathcal{S} \rightarrow \mathcal{S}$ as follows: for every $\boldsymbol{u} \in \mathcal{S}$, draw a line of support to $\mathcal{S}$ at the point $\boldsymbol{u}$ closest to the point $\boldsymbol{a}$. Denote this straight line by $\ell(\boldsymbol{u})$. Then define $\varphi(\boldsymbol{u})$ as the point symmetric (with respect to the bisector) to the pole $\boldsymbol{v}$ of the line $\ell(\boldsymbol{u})$ (Fig. 7).

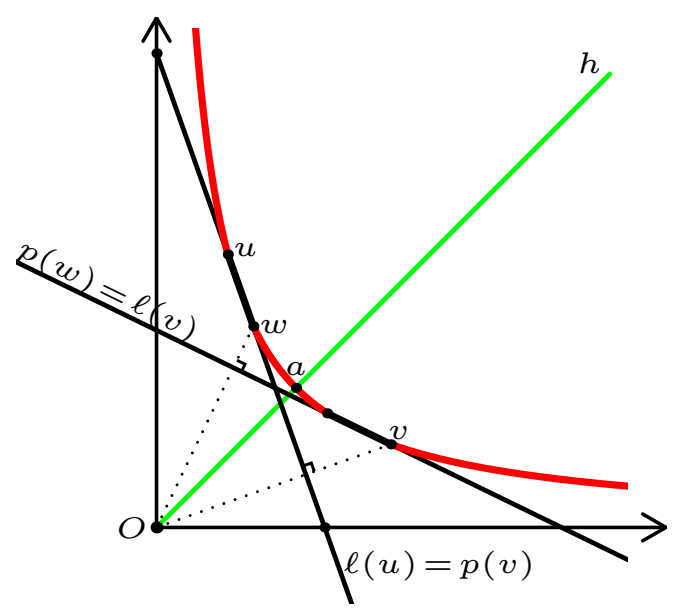

Figure 7: Proof of Theorem 5. The non-smooth case.

Clearly, if $\mathcal{S}$ has an edge, then all points $\boldsymbol{u}$ of that edge, except for its end closest to $\boldsymbol{a}$, have the same image $\varphi(\boldsymbol{u})$. Indeed, for all such points $\boldsymbol{u}$, the line $\ell(\boldsymbol{u})$ is the same. Therefore it suffices to consider the case when $\boldsymbol{u}$ is the end of the edge most distant from $\boldsymbol{a}$. Denote by $\boldsymbol{w}$ the other end of that edge. If $\boldsymbol{w}=\boldsymbol{u}$, then the edge $[\boldsymbol{u}, \boldsymbol{w}]$ is trivial and $\mathcal{S}$ is smooth at the point $\boldsymbol{u}$. Since $f$ is self-dual, we have $\boldsymbol{v} \in \mathcal{S}$ and the set of polars to all $\boldsymbol{x} \in[\boldsymbol{u}, \boldsymbol{w}]$ is the set of lines of support to $\mathcal{S}$ at the point $\boldsymbol{v}$. Among them, the polar to $\boldsymbol{w}$ is the closest one to $\boldsymbol{a}$. Hence, the pole of the line $\ell(\boldsymbol{v})$ is $\boldsymbol{w}$ and consequently $\varphi(\boldsymbol{v})=\boldsymbol{w}^{\prime}$, where $\boldsymbol{w}^{\prime}, \boldsymbol{u}^{\prime}, \boldsymbol{v}^{\prime}$ are the points symmetric to $\boldsymbol{w}, \boldsymbol{u}$, and $\boldsymbol{v}$ respectively about the bisector of the coordinate angle. Since $\mathcal{S}$ is symmetric, it contains all $\boldsymbol{w}^{\prime}, \boldsymbol{u}^{\prime}$, and $\boldsymbol{v}^{\prime}$ and $\varphi\left(\boldsymbol{v}^{\prime}\right)=\boldsymbol{w}$. Taking into account that $\boldsymbol{v}^{\prime}=\varphi(\boldsymbol{u})$, we conclude that $\varphi(\varphi(\boldsymbol{u}))=\boldsymbol{w}$. In particular, if $f$ is smooth at $\boldsymbol{u}$, then $\varphi(\varphi(\boldsymbol{u}))=\boldsymbol{u}$.

Now consider the natural parametrisation of $\mathcal{S}_{1}$ and the corresponding function $\varphi: \mathbb{R}_{+} \rightarrow$ 
$\mathbb{R}_{+}$. It satisfies all the assumptions of Lemma 3 with the set $\Omega$ being the image of the union of nontrivial half-open edges of $\mathcal{S}_{1}$ (we remove the end closest to $\boldsymbol{a}$ from each edge). Applying Lemma 3 we obtain $\varphi(\boldsymbol{u})=\boldsymbol{w}$. Hence $\boldsymbol{w}$ is symmetric to $\boldsymbol{v}$. Consider the right triangle $\boldsymbol{b} O \boldsymbol{c}$ formed by the line $\ell(\boldsymbol{u})$ and by the two coordinate axes. As in the proof for the smooth case we conclude that $\boldsymbol{w}$ is the midpoint of the hypotenuse $\boldsymbol{b} \boldsymbol{c}$ and that the area of $\boldsymbol{b} O \boldsymbol{c}$ is $2 x y$, where $\boldsymbol{w}=(x, y)$ (Fig. 7). This is well known that in this case the line $\boldsymbol{a} \boldsymbol{b}$ touches the hyperbola $\mathcal{H}=\left\{(x, y) \in \mathbb{R}_{+}^{2} \mid 2 x y=1\right\}$ at the point $\boldsymbol{w}$. Actually, $\mathcal{H}$ is not the whole hyperbola, but only one of two branches, but we keep a short notation.

Thus, we have proved the following property of the curve $\mathcal{S}$ : each edge of this curve is tangent to the hyperbola $\mathcal{H}$ at its end closest to the point $\boldsymbol{a}$ (Fig. 7). In particular, all points of $\mathcal{S}$ out of nontrivial edges belong to $\mathcal{S}$. This implies that $\mathcal{S}=\mathcal{H}$. Indeed, if $\mathcal{S}$ has a proper edge $[\boldsymbol{u}, \boldsymbol{w}]$, then it touches $\mathcal{H}$ at the point $\boldsymbol{w}$ and hence $\boldsymbol{u} \notin \mathcal{H}$. Therefore, there exists a point $\boldsymbol{x} \in \mathcal{S}$ close to $\boldsymbol{u}$ such that the arc of $\mathcal{S}$ between the points $\boldsymbol{u}$ and $\boldsymbol{x}$ intersects neither $\mathcal{H}$ nor the half-open interval $(\boldsymbol{u}, \boldsymbol{w}]$. Since $\boldsymbol{x}$ does not lie on $\mathcal{H}$, it must belong to a proper edge $[\boldsymbol{m}, \boldsymbol{n}]$. In this case $\boldsymbol{n} \in \mathcal{H}$, which is impossible, since $\boldsymbol{n}$ belongs to the arc between $\boldsymbol{u}$ and $\boldsymbol{x}$, which does not intersect $\mathcal{H}$. The contradiction proves that $\mathcal{S}$ does not have proper edges, therefore all its points belong to $\mathcal{H}$ and so $\mathcal{S}=\mathcal{H}$.

Theorem 5 implies that the only autopolar symmetric conic body in $\mathbb{R}_{+}^{2}$ is the hyperbola $\sqrt{2 x y}=1$. Possible generalizations of this result to $\mathbb{R}_{+}^{d}$ are discussed in the next section.

\section{Open problems}

As shown in Section 4, there are infinitely many self-dual antinorms in $\mathbb{R}_{+}^{d}$. However, we succeed in their classifying only for $d=2$. So, the first open problem is the following:

Problem 1. How to characterise self-dual antinorms in $\mathbb{R}_{+}^{d}$ for $d \geq 3$ ?

In the two-dimensional case, every self-dual antinorm is constructed starting with the vector $\boldsymbol{a}$ (see Proposition 6). Draw the ray $h=\{t \boldsymbol{a} \mid t \geq 0\}$ and choose arbitrary mutually dual antinorms in those parts. In the $d$-dimensional case, Proposition 6 still holds and we can draw a ray $h$, but it does not split the positive orthant $\mathbb{R}_{+}^{d}$. Most likely, constructing self-dual antinorms for $d \geq 3$ requires other ideas.

The situation with polyhedral self-dual antinorms is still more complicated. As for now, we are not only able to classify them for $d \geq 3$ but do not know if they exist at all, apart from liftings of two-dimensional antinorms. The lifting is defined as follows. Let $P$ be a $k$-dimensional conic polyhedron in some of $k$-dimensional faces of $\mathbb{R}_{+}^{d}$, say, in the face consisting of points $\left(x_{1}, \ldots, x_{k}, 0, \ldots, 0\right)$. The lifting of $P$ in $\mathbb{R}^{d}$ is $G=\left\{\left(x_{1}, \ldots, x_{d}\right) \in\right.$ $\left.\mathbb{R}_{+}^{d} \mid\left(x_{1}, \ldots, x_{k}\right) \in P\right\}$. Clearly, $G$ is also a conic polyhedron, which is the right cylinder 
with the base $P$. Moreover, if $P$ is autopolar, then so is $G$. If $P$ is a unit ball of the antinorm $\varphi\left(x_{1}, \ldots, x_{k}\right)$ on the corresponding face of $\mathbb{R}_{+}^{d}$, then $G$ is a unit ball of the antinorm $f(\boldsymbol{x})=\varphi\left(x_{1}, \ldots, x_{x}\right)$ on $\mathbb{R}_{+}^{d}$.

Each autopolar conic polygon in $\mathbb{R}_{+}^{2}$ produces an autopolar conic polyhedron in $\mathbb{R}_{+}^{d}$ by the lifting. Hence, there are infinitely many $d$-dimensional conic polyhedra. The question is if there are others?

Problem 2. Does there exist at least one autopolar conic polyhedron in $\mathbb{R}^{3}$, which is not a lifting of a smaller-dimensional conic polyhedron? The same question is for higher dimensions.

In two-dimensional case we built autopolar conic polygons starting with the point $\boldsymbol{a}$ (the closest point to the origin) and then constructing successively other vertices, see subsection 4.3. In higher dimensions, however, this approach does not seem to be applicable.

Our last problem concerns the symmetric antinorms.

Problem 3. Is it true that $f(\boldsymbol{x})=\sqrt{d}\left(x_{1} \cdots x_{d}\right)^{1 / d}$ is the unique self-dual symmetric antinorm in $\mathbb{R}_{+}^{d}$ ?

For $d=2$, the affirmative answer is given in Theorem 5. Its proof was based on the general construction of two-dimensional self-dual antinorms, which is not applicable in higher dimensions. It is interesting whether the proof of the $d$-dimensional analogue of Theorem 5 , provided it is correct, can be derived by applying Theorem 5 and a kind of inductive argument?

\section{Applications}

We consider several applications of antinorms: the linear switching systems, the largest Lyapunov exponent, the lower spectral radius of matrices, and the convex trigonometry. We will see that the role and the interpretation of duality and of self-duality is different in those applications.

\subsection{Lyapunov functions for linear switching systems}

The linear switching system is a dynamical system defined for an arbitrary compact set of $d \times d$ matrices $\mathcal{A}$ (the control set) as follows:

$$
\left\{\begin{array}{l}
\dot{\boldsymbol{x}}(t)=A(t) \boldsymbol{x}(t), \quad t \geq 0 \\
\boldsymbol{x}(0)=\boldsymbol{x}_{0} \\
A(t) \in \mathcal{A}, \quad t \geq 0
\end{array}\right.
$$

The function $A(\cdot)$ called the switching law is a measurable function from $\mathbb{R}_{+}$to the control set of matrices $\mathcal{A}$. Thus, we have a linear differential equation with the controlled matrix $A(\cdot)$ in the right hand side. 
See [20, 28] for general theory and applications of linear switching systems. One of important issues is estimating the fastest possible growth of trajectories $\boldsymbol{x}(t)$ as $t \rightarrow \infty$. A measure of the fastest growth $\hat{\sigma}(\mathcal{A})$ is equal to the infimum of numbers $\alpha$ such that $\|\boldsymbol{x}(t)\| \leq C e^{\alpha t}$ for every trajectory $\boldsymbol{x}(t)$. The system is (asymptotically) stable if every trajectory tends to zero as $t \rightarrow+\infty$. The stability is equivalent to the condition $\hat{\sigma}(\mathcal{A})<0[27$. The standard way to prove the stability is to find a Lyapunov function, which is a continuous positive homogeneous function on $\mathbb{R}^{d}$ that decreases along every trajectory. The existence of a Lyapunov function implies the stability. The converse is also true. Moreover, each stable system possesses a convex Lyapunov function, i.e., a norm decreasing along every trajectory [27]. The most popular Lyapunov functions (quadratic, piecewise-quadratic, polyhedral, sum-of-squares, etc.) are norms. If the control set $\mathcal{A}$ is convex, then there exists an invariant norm also called Barabanov norm $\|\cdot\|$ such that for every trajectory, we have $\|\boldsymbol{x}(t)\| \leq e^{\hat{\sigma}}\|\boldsymbol{x}(0)\|, t \geq 0$, and for every point $\boldsymbol{x}_{0}$, there exists a trajectory $\overline{\boldsymbol{x}}(t)$ starting at that point and such that $\|\boldsymbol{x}(t)\|=e^{\hat{\sigma}}\|\boldsymbol{x}(0)\|, t \geq 0$ [1]. Apart from some special cases, it is not known how to find invariant norms. Nevertheless, constructing "nearly invariant" norms makes it possible to compute $\hat{\sigma}$ with a good precision [2, 13, 21, 28].

Similarly, the slowest growth of trajectories of linear switching systems is characterized by the value $\check{\sigma}(\mathcal{A})$ equal to the infimum of numbers $\alpha$, for which there exists a control function $A(\cdot)$ such that for every starting point $\boldsymbol{x}_{0} \neq 0$, the corresponding trajectory satisfies $\|\boldsymbol{x}(t)\| \leq C e^{\alpha t}$. The system is stabilizable if there is a control function $A(\cdot)$ such that every trajectory with the switching law $A(\cdot)$ tends to zero as $t \rightarrow+\infty$ independently of the initial point $\boldsymbol{x}_{0}$. The stabilizability is equivalent to the condition $\check{\sigma}<0$ [21, 39]. To decide the stabilizability one can consider a homogeneous positive Lyapunov function $f: \mathbb{R}^{d} \rightarrow \mathbb{R}_{+}$ which increases along every trajectory. If such a function exists, then clearly the system is not stabilizable. However, the converse is in general not true: for non stabilizable systems, such a function may not exist [2]. The reason is that here we cannot rely on convexity. All known proofs of the existence of the Lyapunov norm for stable systems [1, 41] use the fact that the pointwise supremum of convex functions is also convex. For stabilizable systems, one needs to use infinum instead of supremum, but for infimum this property does not hold: the pointwise infimum does not respect convexity. One could then replace the convexity by concavity. However, there are no concave positive functions on $\mathbb{R}^{d}$ apart from identical constants. Nevertheless, such functions exist on cones. In fact, if the system has an invariant cone, then there always exists a Lyapunov concave function (antinorm) on that cone. This is true, in particular, for positive systems that have their trajectories in the positive orthant $\mathbb{R}_{+}^{d}$. Moreover, a positive system always possesses an invariant antinorm $f$ such that for every trajectory, we have $f(\boldsymbol{x}(t)) \geq e^{\check{\sigma}} f(\boldsymbol{x}(0)), t \geq 0$, and for every point $\boldsymbol{x}_{0} \in \mathbb{R}_{+}^{d}$, there exists a trajectory $\overline{\boldsymbol{x}}(t)$ starting at that point and such that $f(\overline{\boldsymbol{x}}(t))=e^{\check{\sigma}} f(\overline{\boldsymbol{x}}(0))$ for all $t \geq 0$ [13]. Stability and stabilizabilty of positive systems have been studied in many works [8, 13] and references therein.

Approximating the invariant antinorm makes it possible to compute the Lyapunov exponent $\hat{\sigma}$ with a prescribed precision. Duality of antinorms enables us to find the following relations between the systems with control sets $\mathcal{A}$ and $\mathcal{A}^{T}=\left\{A^{T} \mid A \in \mathcal{A}\right\}$. 
Proposition 7 If $f$ is a Lyapunov antinorm for the system with a control set $\mathcal{A}$, then $f^{*}$ is a Lyapunov antinorm for the system with the control set $\mathcal{A}^{T}$.

Remark 7 Proposition 7 is not that obvious as it seems. For example, it is not true for invariant antinorms: if $f$ is an invariant antinorm for $\mathcal{A}$, then $f^{*}$ is, in general, not an invariant antinorm for $\mathcal{A}^{T}$

Proof of Proposition 7. If $f$ is a Lyapunov antinorm, then for every sufficiently small $s>0$, there exists $\varepsilon=\varepsilon(s)>0$ such that $f(\boldsymbol{x}+s A \boldsymbol{x})<(1-\varepsilon) f(\boldsymbol{x})$ for every $\boldsymbol{x}>0$ and $A \in \mathcal{A}[13]$. Then, for every $\boldsymbol{y}>0$, we have

$$
f^{*}\left(\boldsymbol{y}-s A^{T} \boldsymbol{y}\right)=\inf _{\boldsymbol{x}>0} \frac{\left(\boldsymbol{x},\left(I-s A^{T}\right) \boldsymbol{y}\right)}{f(\boldsymbol{x})}=\inf _{\boldsymbol{x}>0} \frac{((I-s A) \boldsymbol{x}, \boldsymbol{y})}{f(\boldsymbol{x})} \leq \inf _{\boldsymbol{x}>0} \frac{((I-s A) \boldsymbol{x}, \boldsymbol{y})}{f(\boldsymbol{x})} .
$$

Denote $\boldsymbol{z}=(I-s A) \boldsymbol{x}$. Then $\boldsymbol{x}=(I-s A)^{-1} \boldsymbol{z}=(I+s A+o(s)) \boldsymbol{z}$ as $s \rightarrow 0$. Hence, for sufficiently small $S$, we have

$f^{*}\left(\boldsymbol{y}-s A^{T} \boldsymbol{y}\right)=\inf _{\boldsymbol{z}>0} \frac{(\boldsymbol{z}, \boldsymbol{y})}{f(\boldsymbol{z}+s A \boldsymbol{z}+o(s))}>\inf _{\boldsymbol{z}>0} \frac{(\boldsymbol{z}, \boldsymbol{y})}{(1-\varepsilon) f(\boldsymbol{z})}=\frac{1}{1-\varepsilon} f^{*}(\boldsymbol{y})>(1+\varepsilon) f^{*}(\boldsymbol{y})$.

Thus, $f^{*}\left(\boldsymbol{y}-s A^{T} \boldsymbol{y}\right)>(1+\varepsilon) f^{*}(\boldsymbol{y})$ for every $A^{T} \in \mathcal{A}^{T}$, therefore $f^{*}$ is a Lyapunov antinorm for the family $\mathcal{A}^{T}$, see [13].

Proposition 7 establishes the duality of Lyapunov antinorms for transposed families. In practice, this allows us to construct the Lyapunov function by passing to the transpose family, for which this problem is sometimes simpler.

\subsection{The largest Lyapunov exponent of matrices}

The antinorm is also applied for computation of the largest Lyapunov exponent arising in the multiplicative ergodic theorem. For the sake of simplicity, we consider the case of products of independent random matrices distributed over a finite matrix family. Let us have a family of $d \times d$-matrices $\mathcal{A}=\left\{A_{1}, \ldots, A_{m}\right\}$. To each matrix $A_{j}$ we associate a positive probability $p_{j}$ so that $\sum_{j=1}^{m} p_{j}=1$. Consider a random product $X_{k}=A_{d_{k}} \cdots A_{d_{1}}$, where all indices $\left\{d_{j}\right\}$ are independent and identically distributed random variables; each $d_{j}$ takes values $1, \ldots, m$ with probabilities $p_{1}, \ldots, p_{m}$ respectively. According to the FurstenbergKesten theorem [11] the value $\left\|X_{k}\right\|^{1 / k}$ converges with probability 1 to a number $r$, which depends only on the family $\mathcal{A}$, and on the probabilities $\left\{p_{j}\right\}_{j=1}^{m}$. The number $\ell=\log r$ is called the largest Lyapunov exponent of $\mathcal{A}$. A strong generalization of this theorem was proved by Oseledets [31]. We do not deal with other Lyapunov exponents, and omit the word "largest". This, however, should not lead to a confusion with the Lyapunov exponent 
for the linear switching system (from the previous section). This number can be found by the following limit formula

$$
\ell=\lim _{k \rightarrow \infty} \frac{1}{k} \boldsymbol{E} \log \left\|A_{d_{k}} \cdots A_{d_{1}}\right\|
$$

where $\boldsymbol{E}$ denotes the mathematical expectation. The computation of the Lyapunov exponent is hard even for $2 \times 2$ matrices. No efficient algorithms are known. This is not surprising because the Lyapunov exponent is in general a discontinuous function of matrices. Moreover, the problem to distinguish between two cases: $\ell \geq 0$ and $\ell<0$ is algorithmically undecidable [3]. For nonnegative matrices, the situation is slightly better. In this case the Lyapunov exponent is continuous, and there are numerically efficient algorithm for its approximate computation, see, for instance [12, 18, 33] and references therein. Although the distinguishing between $\ell \geq 0$ and $\ell<0$ is NP-hard even for Boolean matrices [3]. For properties of Lyapunov exponents of non-negative matrices, see [12, 36, 40].

Some of the computational algorithms are based on approximation of the Lyapunov antinorm $f$ on $\mathbb{R}_{+}^{d}$, which is characterised by the property:

$$
\prod_{j=1}^{m} f^{p_{j}}\left(A_{j} \boldsymbol{x}\right)<f(\boldsymbol{x}), \quad \boldsymbol{x} \in \mathbb{R}_{+}^{d} .
$$

Under some mild assumption on matrices (the absence of zero rows and columns and of common invariant coordinate subspaces), the following holds: $\ell<0$ if and only if there exists a strictly positive Lyapunov antinorm [34. Moreover, under the same assumptions, there always exists an invariant antinorm, for which

$$
\prod_{j=1}^{m} f^{p_{j}}\left(A_{j} \boldsymbol{x}\right)=r f(\boldsymbol{x}), \quad \boldsymbol{x} \in \mathbb{R}_{+}^{d},
$$

see [35]. Surprisingly enough, an analogue of Proposition 7 is not true for the random matrix products: the dual to a Lyapunov antinorm of a matrix family $\mathcal{A}$ may not be a Lyapunov antinorm for the transpose family $\mathcal{A}^{T}$.

Proposition 8 There exists a family $\mathcal{A}$ of nonnegative $2 \times 2$ matrices and an antinorm $f$ on $\mathbb{R}_{+}^{2}$ such that $f$ is a Lyapunov function for $\mathcal{A}$, but $f^{*}$ is not a Lyapunov function for $\mathcal{A}^{T}$.

Proof. Take an arbitrary $q \in\left(\frac{\sqrt{2}}{2}, 1\right)$ and consider the following pair of matrices:

$$
A_{1}=q\left(\begin{array}{cc}
1 & 1 \\
0 & 1
\end{array}\right) ; \quad A_{2}=q\left(\begin{array}{cc}
1 & 0 \\
1 & 1
\end{array}\right)
$$

with the probabilities $p_{1}=p_{2}=\frac{1}{2}$. Since $A_{2}=A_{1}^{T}$, the family $\mathcal{A}=\left\{A_{1}, A_{2}\right\}$ satisfies $\mathcal{A}=\mathcal{A}^{T}$. Consider the antinorm $f(x, y)=x+y$ on $\mathbb{R}_{+}^{2}$. Then its dual is $f^{*}(x, y)=$ $\min \{x, y\}$ (see Example 3). For each point $\boldsymbol{x}=(x, y)$ such that $f(x, y)=1$, we have 
$A_{1} \boldsymbol{x}=q(x+y, y)^{T}=q(1, y)^{T}$. Hence, $f\left(A_{1} \boldsymbol{x}\right)=q(1+y)$. Similarly, $A_{2} \boldsymbol{x}=q(x, 1)^{T}$ and $f\left(A_{2} \boldsymbol{x}\right)=q(1+x)$. Therefore,

$$
f^{p_{1}}\left(A_{1} \boldsymbol{x}\right) f^{p_{2}}\left(A_{2} \boldsymbol{x}\right)=\sqrt{f\left(A_{1} \boldsymbol{x}\right) f\left(A_{2} \boldsymbol{x}\right)}=q \sqrt{(1+y)(1+x)} \geq q \sqrt{2}>1=f(\boldsymbol{x})
$$

Thus, for every $\boldsymbol{x} \in \mathbb{R}_{+}^{2}$, we have $f^{p_{1}}\left(A_{1} \boldsymbol{x}\right) f^{p_{2}}\left(A_{2} \boldsymbol{x}\right)>f(\boldsymbol{x})$, hence $f$ is a Luapunov antinorm for $\mathcal{A}$.

On the other hand, if $f *(\boldsymbol{x})=\min \{x, y\}=1$, then $f^{*}\left(A_{1} \boldsymbol{x}\right)=\min \{q(x+y), q y\}=q y$ and $f^{*}\left(A_{2} \boldsymbol{x}\right)=\min \{q x, q(x+y)\}=q x$, consequently $\sqrt{f^{*}\left(A_{1} \boldsymbol{x}\right) f^{*}\left(A_{2} \boldsymbol{x}\right)}=q \sqrt{x y}$. At the point $\boldsymbol{x}=(1,1)$, we have $\sqrt{f^{*}\left(A_{1} \boldsymbol{x}\right) f^{*}\left(A_{2} \boldsymbol{x}\right)}=q<1$, and so $\sqrt{f^{*}\left(A_{1} \boldsymbol{x}\right) f^{*}\left(A_{2} \boldsymbol{x}\right)}<f^{*}(\boldsymbol{x})$, therefore, $f^{*}$ is not a Lyapunov antinorm for $\mathcal{A}$, neither for $\mathcal{A}^{T}=\mathcal{A}$.

Thus, dual antinorms may not correspond to the Lyapunov antinorms of transposed families of matrices. It would be interesting to understand the sense of duality for the Lyapunov antinorms.

\subsection{The lower spectral radius}

The lower spectral radius (also called in the literature the joint spectral subradius) of a compact family of matrices $\mathcal{A}$ is

$$
\check{\rho}(\mathcal{A})=\lim _{k \rightarrow \infty} \min _{d_{1}, \ldots, d_{k}}\left\|A_{d_{k}} \cdots A_{d_{1}}\right\|^{1 / k},
$$

where the minimum is defined over all possible products of length $k$ of matrices from $\mathcal{A}$, with repetitions permitted. This limit always exists and does not depend on the matrix norm. For a family of one matrix, the lower spectral radius becomes the usual spectral radius of that matrix, which is the maximum modulus of its eigenvalues. The lower spectral radius is the exponent of the minimal growth of matrix products of length $k$ as $k \rightarrow \infty$.

The lower spectral radius was introduced in [17] to characterise the minimal growth of trajectories of the discrete-time linear switching system $\boldsymbol{x}(k+1)=A(k) \boldsymbol{x}(k)$ as $k \rightarrow \infty$, where $A(k) \in \mathcal{A}, k \geq 0$. The sequence $A(k)$ is called the switching law. The system is stabilizable if there exists a switching law whose trajectory $\{\boldsymbol{x}(k)\}_{k \geq 0}$ tends to zero as $k \rightarrow \infty$, for every initial point $\boldsymbol{x}(0) \in \mathbb{R}^{d}$. The stabilizability is equivalent to the condition $\check{\rho}(\mathcal{A})<1$. See [4, 14, 29] for more properties of the lower spectral radius. Apart from the dynamical systems, it has found applications in the theory of wavelets, in approximation theory, in the number theory, combinatorics, the theory of formal languages, etc.

Many of those applications (see, for example, [9, 10, 14, 19, 37]) deal with nonnegative matrix families. For them, the lower spectral radius can be efficiently bounded in terms of antinorms. For an arbitrary nonnegative family $\mathcal{A}$, there exists an extremal antinorm $f$ on $\mathbb{R}_{+}^{d}$ such that

$$
\min _{A \in \mathcal{A}} f(A \boldsymbol{x}) \geq \check{\rho}(\mathcal{A}) f(\boldsymbol{x}) \quad \boldsymbol{x} \in \mathbb{R}_{+}^{d} .
$$

Moreover, under some mild assumptions on $\mathcal{A}$, there is also an invariant antinorm [14, Theorems 5,6]: 
Definition 4 An antinorm $f$ on $\mathbb{R}_{+}^{d}$ is called invariant for a family $\mathcal{A}$ of non-negative $d \times d$ matrices if

$$
\min _{A \in \mathcal{A}} f(A \boldsymbol{x})=\check{\rho}(\mathcal{A}) f(\boldsymbol{x}) \quad \boldsymbol{x} \in \mathbb{R}_{+}^{d} .
$$

Efficient algorithms for approximate computation of the lower spectral radius are based on the construction of extremal and invariant antinorms [14]. Those antinorms are also of an independent interest [15].

The following theorem establishes a relation between the extremal antinorms of a matrix family and of its transpose. Thus, for the lower spectral radius, this relation is found, unlike for the Lyapunov exponent of random matrix products (Proposition 8). Moreover, it is also possible to characterize not only the extremal antinorm, but also the invariant antinorm of a transpose family, in contrast to the situation with the continuous-time switching systems (Remark 7). To formulate the theorem we need to introduce some more notation. For an arbitrary subset $X$ of $\mathbb{R}_{+}^{d}$, we consider its positive convex hull $\mathrm{co}_{+} X=$ co $X+\mathbb{R}_{+}^{d}=\left\{\boldsymbol{x} \in \mathbb{R}_{+}^{d} \mid \exists \boldsymbol{y} \in \operatorname{co} X\right\}$, where, recall, co $X$ is the (standard) convex hull of $X$. A conic body $P \subset \mathbb{R}_{+}^{d}$ is called invariant for a family $\mathcal{A}$ of nonnegative matrices if $\mathrm{CO}_{+}\{A P \mid A \in \mathcal{A}\}=\check{\rho} P$.

Theorem 6 If an antinorm $f$ is extremal for $\mathcal{A}$, then $f^{*}$ is extremal for $\mathcal{A}^{T}$. If an antinorm $f$ is invariant for $\mathcal{A}$, then the polar $G^{*}$ to its unit ball is an invariant conic body for $\mathcal{A}^{T}$, and vice versa: if $G$ is an invariant conic body for $\mathcal{A}$, then the antinorm with the unit ball $G^{*}$ is invariant for $\mathcal{A}^{T}$.

Proof. Without loss of generality it can be assumed that $\check{\rho}(\mathcal{A})=1$. If $f$ is an extremal antinorm for $\mathcal{A}$, then

$$
\min _{\boldsymbol{x} \geq 0, f(\boldsymbol{x}) \geq 1, A \in \mathcal{A}} f(A \boldsymbol{x})=1 .
$$

For the dual antinorm $f^{*}$, we have $\min _{\boldsymbol{p} \geq 0, f^{*}(\boldsymbol{p}) \geq 1, A \in \mathcal{A}} f^{*}\left(A^{T} \boldsymbol{p}\right)=$

$$
\min _{\boldsymbol{p} \geq 0, f^{*}(\boldsymbol{p}) \geq 1, A \in \mathcal{A}} \min _{\boldsymbol{x} \geq 0, f(\boldsymbol{x}) \geq 1}\left(A^{T} \boldsymbol{p}, \boldsymbol{x}\right)=\min _{\boldsymbol{x}, \boldsymbol{p} \geq 0, f(\boldsymbol{x}) \geq 1, f^{*}(\boldsymbol{p}) \geq 1, A \in \mathcal{A}}(\boldsymbol{p}, A \boldsymbol{x}) .
$$

Applying (15), and replacing $\boldsymbol{y}=A \boldsymbol{x}$, we see that the last expression in $(16)$ is equal to

$$
\begin{aligned}
& \min _{\boldsymbol{x}, \boldsymbol{p} \geq 0, f(A \boldsymbol{x}) \geq 1, f^{*}(\boldsymbol{p}) \geq 1, A \in \mathcal{A}}(\boldsymbol{p}, A \boldsymbol{x})=\min _{\boldsymbol{y}, \boldsymbol{p} \geq 0, f(\boldsymbol{y}) \geq 1, f^{*}(\boldsymbol{p}) \geq 1}(\boldsymbol{p}, \boldsymbol{y})= \\
& \min _{\boldsymbol{p} \geq 0, f^{*}(\boldsymbol{p}) \geq 1} \min _{\boldsymbol{y} \geq 0, f^{*}(\boldsymbol{y}) \geq 1}(\boldsymbol{p}, \boldsymbol{y})=\min _{\boldsymbol{p} \geq 0, f^{*}(\boldsymbol{p}) \geq 1} f^{*}(\boldsymbol{p})=1 .
\end{aligned}
$$

Thus, $\min _{\boldsymbol{p} \geq 0, f^{*}(\boldsymbol{p}) \geq 1, A^{T} \in \mathcal{A}^{T}} f^{*}\left(A^{T} \boldsymbol{p}\right)=1$, hence, $f^{*}$ is an extremal antinorm for $\mathcal{A}^{T}$, which completes the proof of the first statement.

Now we need to prove that if $f$ is an invariant antinorm for $\mathcal{A}$, then $G^{*}$ in an invariant conic body for $\mathcal{A}^{T}$, and vice versa. We prove only the primal assertion, after which the dual assertion becomes obvious. If $f$ is an invariant antinorm for $\mathcal{A}$, then, by definition, for 
every $\boldsymbol{x} \in \mathbb{R}_{+}^{d}$, we have $\min _{A \in \mathcal{A}} f(A \boldsymbol{x})=f(\boldsymbol{x})$. Moreover, the function of support of the set $A^{T} G^{*}$ at the point $\boldsymbol{x}$ is equal to $f(A \boldsymbol{x})$. Indeed,

$$
\min _{\boldsymbol{y} \in A^{T} G^{*}}(\boldsymbol{x}, \boldsymbol{y})=\min _{\boldsymbol{p} \in G^{*}}\left(\boldsymbol{x}, A^{T} \boldsymbol{p}\right)=\min _{\boldsymbol{p} \in G^{*}}(A \boldsymbol{x}, \boldsymbol{p})=f^{* *}(A \boldsymbol{x})=f(A \boldsymbol{x}),
$$

where the latter equality follows from Corollary 2 and from continuity of $f$. Similarly, the function of support of $G^{*}$ at the point $\boldsymbol{x}$ is equal to $f(\boldsymbol{x})$. Since $\min _{A \in \mathcal{A}} f(A \boldsymbol{x})=f(\boldsymbol{x})$, we see that the minimal function of support of the sets $A^{T} G^{*}$ over all $A \in \mathcal{A}$ is equal to the function of support of $G^{*}$. Hence, $\operatorname{co}_{+}\left\{A^{T} G^{*} \mid A \in \mathcal{A}\right\}=G^{*}$.

Remark 8 It is interesting that while the duality takes an extremal antinorm of matrices to an extremal antinorm of their transposes, it does not do the same with the invariant antinorm. If $f$ is an invariant antinorm for $\mathcal{A}$, then $f^{*}$ is not invariant for $\mathcal{A}^{T}$ but $G^{*}$ is an invariant conic body for $\mathcal{A}^{T}$. For the joint spectral radius, which is responsible for the stability of discrete-time linear switching system, a similar relation between the invariant norm and the invariant body of the transpose family was established in [32].

\subsection{The convex trigonometry}

One more application of the duality and self-duality of antinorms is in the extension of convex trigonometry to the convex hyperbolic functions. The convex trigonometry was developed recently [22] in the study of bivariate optimal control problems. Let us recall the basic construction. For an arbitrary convex body $G \subset \mathbb{R}^{2}$ containing the origin $O$ in its interior, we denote by $\mathcal{S}$ its boundary, by $\mathbb{S}$ its area, and for an arbitrary number $\theta \in[0,2 \mathbb{S}]$, we define by $P_{\theta}$ the point on $\mathcal{S}$ such that the oriented area of the part of $G$ bounded by rays $O X$ and $O P_{\theta}$ is equal to $\frac{1}{2} \theta$. This definition is extended to all $\theta \in \mathbb{R}$ in a standard manner by periodicity. Then by definition $\cos _{G} \theta$ and $\sin _{G} \theta$ are respectively the abscissa and the ordinate of $P_{\theta}$. The standard trigonometric functions correspond to the case when $G$ is a unit disc. It was shown [22] that all basic trigonometric formulas can be generalized to $\cos _{G} \theta$ and $\sin _{G} \theta$. In particular, the identity $\cos ^{2} \theta+\sin ^{2} \theta=1$, becomes $\cos _{G} \theta \cos _{G^{*}} \theta^{*}+\sin _{G} \theta \sin _{G^{*}} \theta^{*}=1$, where $G^{*}$ is the polar of $G$ and $\theta^{*}$ corresponds to the point on the boundary of $G^{*}$ defined by the direction of the normal to $G$ drawn at the point $P_{\theta}$.

Thus, every convex body $G \subset \mathbb{R}^{2}$ containing the origin as an interior point defines trigonometric functions. In particular, every norm in $\mathbb{R}^{2}$ defines them by means of its unit ball. It was announced in [22] that a similar construction can produce hyperbolic functions. Indeed, they can be defined by an arbitrary antinorm in $\mathbb{R}_{+}^{2}$ (in this case it should be rather called concave trigonometry). To see this, we consider an antinorm $f$ and the closest to the origin point $P_{0}$ of the unit sphere $\mathcal{S}$ of $f$. Denote by $\tilde{\mathcal{S}}$ the unit sphere $\mathcal{S}$ complemented by 
rays of the coordinate axes in case $\mathcal{S}$ intersects the corresponding axes. Thus, if $f$ vanishes on the axes, then $\tilde{\mathcal{S}}=\mathcal{S}$.

For an arbitrary $\theta \in \mathbb{R}$, we consider the point $P_{\theta} \in \tilde{\mathcal{S}}$ such that the oriented area of the part of the unit ball $G$ bounded by $\tilde{\mathcal{S}}$, by the line $O P_{0}$, and by the perpendicular dropped from $P_{\theta}$ to that line, is equal to $\frac{1}{2} \theta$. By definition $\cosh _{G} \theta$ and $\sinh _{G} \theta$ are respectively the abscissa and the ordinate of $P_{\theta}$. The standard hyperbolic functions correspond to the case when $G$ is the hyperbola $2 x y=1$. The identity $\cosh ^{2} \theta-\sinh ^{2} \theta=1$, becomes $\cosh _{G} \theta \cosh _{G^{*}} \theta^{*}+\sinh _{G} \theta \sinh _{G^{*}} \theta^{*}=1$, where $G^{*}$ is the polar of $G$ and $\theta^{*}$ corresponds to the point on the boundary of $G^{*}$ defined by the direction parallel to the normal to $G$ drawn at the point $P_{\theta}$. If $G$ is autopolar, i.e., $f$ is self-dual, then this formula is simplified to $\cosh _{G} \theta \cosh _{G} \theta^{*}+\sinh _{G} \theta \sinh _{G} \theta^{*}=1$. In contrast to the convex trigonometry, here we have a variety of autopolar sets classified by Theorem 5 . On the other hand, only one of them is symmetric. This is the hyperbola $2 x y=1$ corresponding to the antinorm $f(x, y)=\sqrt{2 x y}$ and producing the standard hyperbolic functions.

Acknowledgements. The author is grateful to the anonymous referee for his impressive work and for many valuable comments. He also expresses his thanks to N.Guglielmi for many useful discussions and to T.Zaitseva for her help in illustrations.

\section{References}

[1] N.E. Barabanov, Absolute characteristic exponent of a class of linear nonstationary systems of differential equations, Siberian Math. J., 29 (1988), 521-530.

[2] F. Blanchini and C. Savorgnanb, Stabilizability of switched linear systems does not imply the existence of convex Lyapunov functions, Automatica, 44 (2008), 1166-1170.

[3] V.D. Blondel and J.N. Tsitsiklis, The Lyapunov exponent and joint spectral radius of pairs of matrices are hard - when not impossible - to compute and to approximate, Math. Control Signals Systems, 10 (1997), 31-40.

[4] J. Bochi and I.D. Morris, Continuity properties of the lower spectral radius, Proc. London Math. Soc., 110 (2014), 477-509.

[5] J.-C. Bourin, F. Hiai, Norm and anti-norm inequalities for positive semi-definite matrices, Internat. J. Math. 22 (2011), 1121-1138.

[6] J.-C. Bourin, F. Hiai, Jensen and Minkowski inequalities for operator means and antinorms, Linear Algebra Appl. 456 (2014), 22-53 
[7] J.-C. Bourin, F. Hiai, Anti-norms on finite von Neumann algebras, Publ. Res. Inst. Math. Sci. 51 (2015), 207-235.

[8] L. Fainshil and M. Margaliot, A maximum principle for the stability analysis of positive bilinear control systems with applications to positive linear switched systems, SIAM J. Control Optim. 50 (2012), 2193-2215.

[9] E. Fornasini and M.E. Valcher, Stability and stabilizability criteria for discrete-time positive switched systems IEEE Trans. Automat. Control 57 (2012), 1208-1221.

[10] E. Fornasini and M.E. Valcher, Asymptotic stability and stabilizability of special classes of discrete-time positive switched systems Linear Algebra Appl. 438 (2013), 1814-1831.

[11] H. Furstenberg and H. Kesten, Products of random matrices, Ann. Math. Statist., 31 (1960), 457-469.

[12] H. Hennion, Limit theorems for products of positive random matrices, Ann. Probab., 25 (1997), 1545-1587.

[13] N. Guglielmi, L. Laglia, and V.Yu. Protasov, Polytope Lyapunov functions for stable and for stabilizable LSS, Found. Comput. Math., 17 (2017), 567-623.

[14] N. Guglielmi and V.Yu. Protasov, Exact computation of joint spectral characteristics of linear operators, Found. Comput. Math., 13 (2013), 37-97.

[15] N. Guglielmi and M.Zennaro, Canonical construction of polytope Barabanov norms and antinorms for sets of matrices, SIAM J. Matrix Anal. Appl. 36 (2015), 634-655.

[16] N. Guglielmi and M. Zennaro, An antinorm theory for sets of matrices: Bounds and approximations to the lower spectral radius, Linear Algebra Appl. 607 (2020), 89-117.

[17] L. Gurvits, Stability of discrete linear inclusions, Linear Algebra Appl., 231 (1995), $47-85$.

[18] R.M. Jungers and V.Yu. Protasov, Lower and upper bounds for the largest Lyapunov exponent of matrices, Linear Algebra Appl., 438 (2013), 4448-4468.

[19] R.M. Jungers, V,Yu. Protasov, and V.D. Blondel, Overlap-free words and spectra of matrices, Theoret. Computer Sci., 410 (2009), 3670-3684.

[20] D. Liberzon, Switching in systems and control, Birkhauser, Boston, MA, 2003. 
[21] H. Lin and P.J. Antsaklis, Stability and stabilizability of switched linear systems: a survey of recent results, IEEE Trans. Autom. Control., 54 (2009), 308-322.

[22] L.V. Lokutsievskiur, Convex trigonometry with applications to sub-Finsler geometry, Sb. Math., 210 (2019), 1179-1205.

[23] H. Martin and K.J. Swanepoel, Antinorms and Radon curves, Aequationes Math. 72 (2006), 110-138.

[24] J.K. Merikoski, On $I_{p 1, p 2}$ antinorms of nonnegative matrices, Linear Algebra Appl. 140 (1990), 31-44.

[25] J.K. Merikoski, On c-norms and c-antinorms on cones, Linear Algebra Appl. 150 (1991), 315-329.

[26] J.K. Merikoski, G. de Oliveira, On k-major norms and k-minor antinorms, Linear Algebra Appl. 176 (1992), 197-209.

[27] A.P. Molchanov and E.S. Pyatnitskii, Lyapunov functions, defining necessary and sufficient conditions for the absolute stability of nonlinear nonstationary control systems, Automat. Remote Control, 47 (1986), 344-354, 443-451, 620-630.

[28] A.P. Molchanov and E.S. Pyatnitskii, Criteria of asymptotic stability of differential and difference inclusions encountered in control theory, System Contr. Letters, 13 (1989), 59-64.

[29] I.D. Morris, Generic properties of the lower spectral radius for some low-rank pairs of matrices, Linear Algebra Appl. 524 (2017), 35-60. .

[30] M. Moszyńska and W-D. Richter, Reverse triangle inequality, antinorms and semiantinorms, Studia Sci. Math. Hungar. 49 (2012), 120-138.

[31] V. I. Oseledets, A multiplicative ergodic theorem. Lyapunov characteristic numbers for dynamical systems, Trans. Moscow Math. Soc., 19 (1968), 197-231.

[32] E. Plischke and F. Wirth, Duality results for the joint spectral radius and transient behaviour, Linear Algebra Appl., 428 (2008), 2368-2384.

[33] M. Pollicott, Maximal Lyapunov exponent for random matrix products, Invent. Math. 181 (2010), 209-226.

[34] V.Yu. Protasov, Invariant functionals of random matrices, Funct. Anal. Appl. 44 (2010), 230-233. 
[35] V.Yu. Protasov, Invariant functionals for the Lyapunov exponents of random matrices, Sb. Math., 202 (2011), 101-126.

[36] V.Yu. Protasov, Asymptotics of products of nonnegative random matrices, Funct. Anal. Appl., 47 (2013), no 2, 138-147.

[37] V.Yu. Protasov, The Euler binary partition function and subdivision schemes, Math. Comp. 86 (2017), 1499-1524.

[38] W.-D. Richter, Convex and radially concave contoured distributions, J. Probab. Stat., 2015, Art. ID 165468, 12 pp.

[39] E. De Santis, M.D. Di Benedetto, G.Pola, Stabilizability of linear switching systems, Nonlinear Anal. Hybrid Syst., 2 (2008), no 3, 750-764.

[40] J.C. Watkins, Limit theorems for products of random matrices: a comparison of two points of view, Contemp. Math., 50 (1986), 5-22.

[41] F. Wirth, The generalized spectral radius and extremal norms, Linear Algebra Appl., 342 (2002), 17-40. 